\title{
Proteomic analysis by iTRAQ-PRM provides integrated insight into mechanisms of resistance in pepper to Bemisia tabaci (Gennadius)
}

Xiaoxia Wu', Jiaxing Yan ${ }^{1}$, Yahong $\mathrm{Wu}^{2}$, Haibo Zhang ${ }^{2}$, Shuangrong $\mathrm{Mo}^{1}$, Xiaoying $\mathrm{Xu}^{1}$, Fucai Zhou ${ }^{1,2^{*}}$ and Haidong Ding ${ }^{1 *}$

\begin{abstract}
Background: The Bemisia tabaci is a major leaf feeding insect pest to pepper (Capsicum annuum), causing serious damage to pepper growth and yield. It is particularly important to study the mechanism of pepper resistance to $B$. tabaci, and to breed and promote the varieties of pepper resistant to B. tabaci. However, very limited molecular mechanism is available about how plants perceive and defend themselves from the destructive pest. Proteome technologies have provided an idea method for studying plant physiological processes in response to $B$. tabaci.

Results: Here, a highly resistant genotype and a highly susceptible genotype were exposed to $B$. tabaci feeding for $48 \mathrm{~h}$ to explore the defense mechanisms of pepper resistance to $B$. tabaci. The proteomic differences between both genotypes were compared using isobaric tag for relative and absolute quantification (iTRAQ). The quantitative data were validated by parallel reaction monitoring (PRM). The results showed that 37 differential abundance proteins (DAPs) were identified in the RG (resistant genotype), while 17 DAPs were identified in the SG (susceptible genotype) at $48 \mathrm{~h}$ after B. tabaci feeding. 77 DAPs were identified when comparing RG with SG without feeding. The DAP functions were determined for the classification of the pathways, mainly involved in redox regulation, stress response, protein metabolism, lipid metabolism and carbon metabolism. Some candidate DAPs are closely related to $B$. tabaci resistance such as annexin D4-like (ANN4), calreticulin-3 (CRT3), heme-binding protein 2-like (HBP1), acidic endochitinase pcht28-like (PR3) and lipoxygenase 2 (LOX2).

Conclusions: Taken together, this study indicates complex resistance-related events in B. tabaci interaction, provides novel insights into the molecular mechanism underlying the response of plant to $B$. tabaci, and identifies some candidate proteins against $B$. tabaci attack.
\end{abstract}

Keywords: Pepper, Bemisia tabaci, Resistance, Proteome, iTRAQ, PRM

\section{Background}

Bemisia tabaci (Gennadius) is one of the most widely distributed agricultural pests that may be harmful to many arable and horticultural crops such as Solanaceae, Cucurbitaceae and Cruciferae. Pepper (Capsicum annuum), an important vegetable type in China, is one of the most serious hosts of B. tabaci. B. tabaci complex

\footnotetext{
* Correspondence: fczhou@yzu.edu.cn; hdding@yzu.edu.cn

'Joint International Research Laboratory of Agriculture and Agri-Product Safety, College of Bioscience and Biotechnology, Yangzhou University, Yangzhou 225009, China

Full list of author information is available at the end of the article
}

contains more than 24 morphologically indistinguishable biotypes [1]. Phylogenetic studies based on mitochondrial cytochrome oxidase I (mtCOI) gene revealed that B. tabaci contains at least 34 putative species [2]. During the past two decades, B. tabaci biotype B has been introduced into at least 54 countries from its origin in the Middle East-Asia Minor region and become a worldwide invasive and destructive whitefly species. In China, B. tabaci was first recorded in the late 1940 s, but was not recorded as the major agricultural pest until the introduction of B. tabaci biotype B in the mid-1990s [3].

(C) The Author(s). 2019 Open Access This article is distributed under the terms of the Creative Commons Attribution 4.0 International License (http://creativecommons.org/licenses/by/4.0/), which permits unrestricted use, distribution, and 
B. tabaci not only damages plants by sucking vital sap from the phloem tissue but also causes indirect damage by vectoring many kinds of viruses and by promoting the growth of saprophytic fungi on the leaves $[4,5]$. The visible, direct damage caused by $B$. tabaci are leaf deformation and honeydew secretion. The control of $B$. tabaci has been relied heavily on the synthetic insecticides, but the pesticide resistance has been developed in many parts of the world. For example, B. tabaci field populations were found highly resistant to imidacloprid and thiamethoxam, both in Israel, Spain, Crete, and China [3]. Plant-insect interactions have resulted in the evolution of sophisticated mechanisms that respond to insect attack [6]. A promising alternative to control $B$. tabaci is to study the resistant mechanism of hostplants, explore resistant genes, and breed for durable host-plant resistance [4, 7]. Therefore, it is particularly important to study the mechanism of pepper resistance to $B$. tabaci, and to breed and promote the varieties of pepper resistant to $B$. tabaci.

Similar to pathogen-plant interaction, B. tabaci induces salicylic acid (SA) defenses and suppresses effectual jasmonic acid (JA) defenses in Arabidopsis [8]. In tomato, $B$. tabaci causes JA levels increase initially and decline within days, whereas the expression of SA-regulated genes was gradually increased $[9,10]$ Feeding by $B$. tabaci is known to induce specific genes such as WFI1 in tomato and $S L W 1$ in squash [11]. Tomato pathogenesis-related protein (PR) genes are also expressed in response to $B$. tabaci biotype B feeding [12]. Recently, RNA-Seq datasets analysis revealed a comprehensive insect resistance response mechanism in cotton to infestation by $B$. tabaci and showed that MPK-WRKY-JA and ethylene (ET) pathways might regulate cotton defenses to $B$. tabaci [13].

Despite these advances, the comprehensive molecular mechanisms underlying plant resistant to $B$. tabaci remain poorly defined. So far, most of these studies focus on nucleic acid level, but fewer have studied the actual protein. Proteome technologies provide an idea method for studying plant physiological processes. Recently, the proteome changes of Arabidopsis thaliana leaves infested by $B$. tabaci were reported using twodimensional electrophoresis and mass spectrometry [6]. Ibrahim et al. [14] showed that the major proteins like MAP kinases, COBRA-like protein family and NBS disease resistance protein were expressed under infested conditions using one-dimensional electrophoresis following liquid chromatography coupled with tandem mass spectrometry (LC-MS/MS). To investigate the pepper-B. tabaci interaction, two genotypes were identified exhibiting different $B$. tabaci susceptibilities, one that was a highly resistant genotype (termed RG) and another that was a highly susceptible genotype (termed SG), and the proteomic differences between both genotypes after B. tabaci infestation for $48 \mathrm{~h}$ were compared using isobaric tag for relative and absolute quantification (iTRAQ). The results may contribute to our understanding of protein response and alteration and provide insights into the molecular mechanisms involved in response to $B$. tabaci infestation in plants.

\section{Results}

\section{Identification of $B$. tabaci resistance in peppers}

In the preliminary experiment, we screened a lot of pepper materials and two pepper genotype varieties were identified showing either high levels of resistance (RG) or susceptibility (SG) to B. tabaci infestation. The resistant characteristics of both genotypes were investigated after $B$. tabaci infestation (Figs. 1 and 2). It is observed that the leaf of RG showed deep green and the leaves of SG looks displayed light green color (Fig. 2 a, b). Besides, different settling behavior of $B$. tabaci adults was found between two varieties. The population of $B$. tabaci settled on SG was about 40 times higher than on RG. Similarly, higher egg hatchability was observed in SG whereas lower egg hatchability was observed in RG (Fig. 2c) Therefore, the two genotypes are ideal candidates for studying the proteomic mechanisms of pepper in response to $B$. tabaci infestation.

\section{iTRAQ protein profiling}

To investigate the mechanisms of pepper resistance against $B$. tabaci, the proteomic profiling analysis at $48 \mathrm{~h}$ after $B$. tabaci treatment of resistant and susceptible genotypes was performed using iTRAQ. The average of the spectral identification rates of the secondary mass spectra produced by the three replicates reached $34 \%$ or more. For example, in the replicate one, a total of 397, 554 spectra were obtained, in which 13,5885 spectra were matched to the known database and the spectral identification rate is $31.2 \%$. A total of 20,102 peptides and 2756 proteins (at least two unique peptides with high confidence) were identified by iTRAQ analysis against the Uniprot database Capsicum annuum (39,809 items) (Additional file 4: Table S1).

The peptide number analysis of the identified proteins showed that the peptide segment numbers in the most of proteins were identified to contain less than 12 (Fig. 3a). The number of proteins containing at least 2 unique peptides in the three batches of this study were 2756, 2658, 3001 , accounting for $79.80,79.56$, and $80.65 \%$ of the total protein, respectively. The percentage of protein with a coverage of $[0,10 \%]$ is $33.66 \%$, the protein with coverage greater than or equal to $20 \%$ accounted for $42.84 \%$ of the total protein, and the average of protein identification coverage was $21.07 \%$ (Fig. 3b). Besides, venn diagram of three batches showed that there were about $80 \%$ shared proteins indicating the high repeatability (Fig. 3c). 


Fig. 1 Strategy for analysis of protein expression in pepper leaves by 4-plex isobaric tagging. SG, susceptible genotype; $\mathrm{RG}$, resistant genotype; SB,
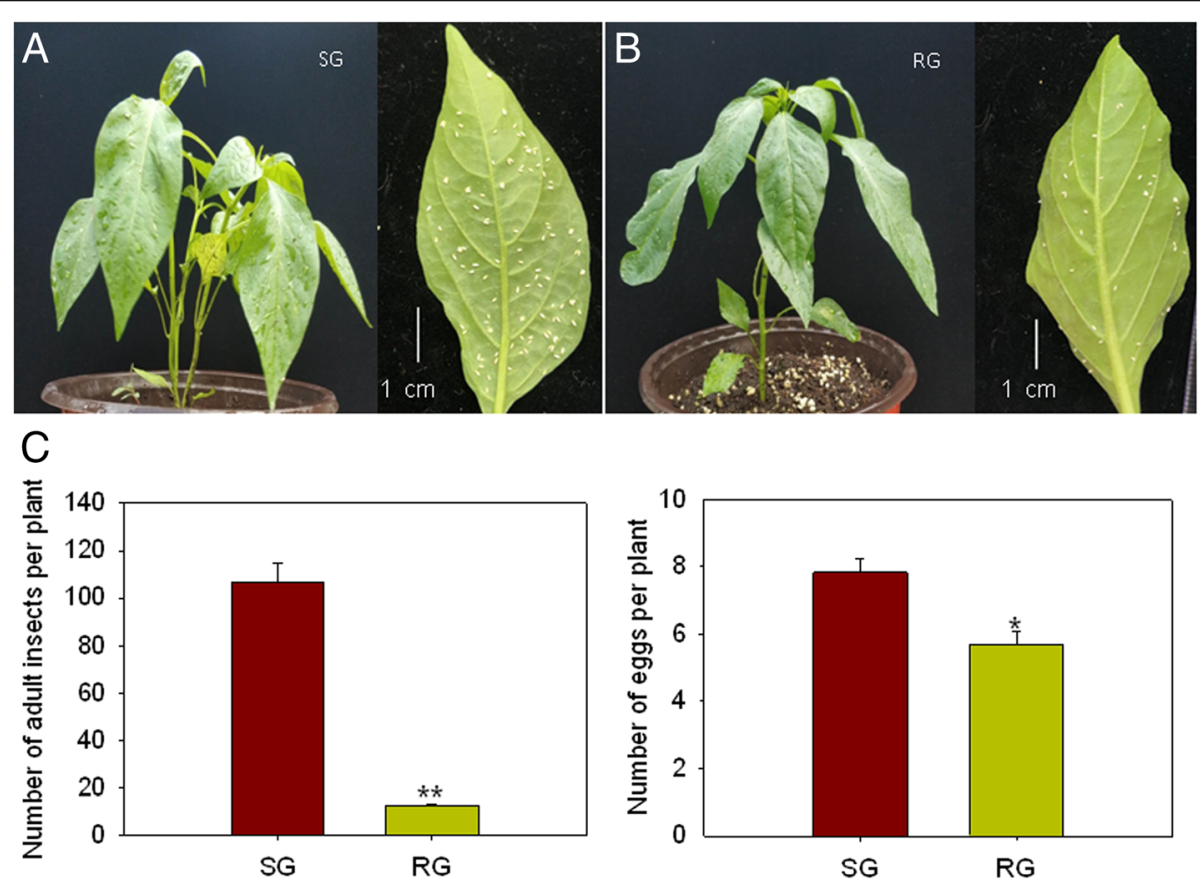

Fig. 2 Symptom of different pepper genotypes, a susceptible genotype (SG) and a resistant genotype (RG) exposed to B. tabaci feeding. a Representative images of the SG following B. tabaci infestation. $\mathbf{b}$ Representative images of the RG following B. tabaci infestation. Left, the plant phenotype; Right, the mature B. tabaci populations on single leaf from either the SG or RG. Plants were infested with B. tabaci in the greenhouse for 72 h. c Anti-selectivity of different pepper varieties on B. tabaci 

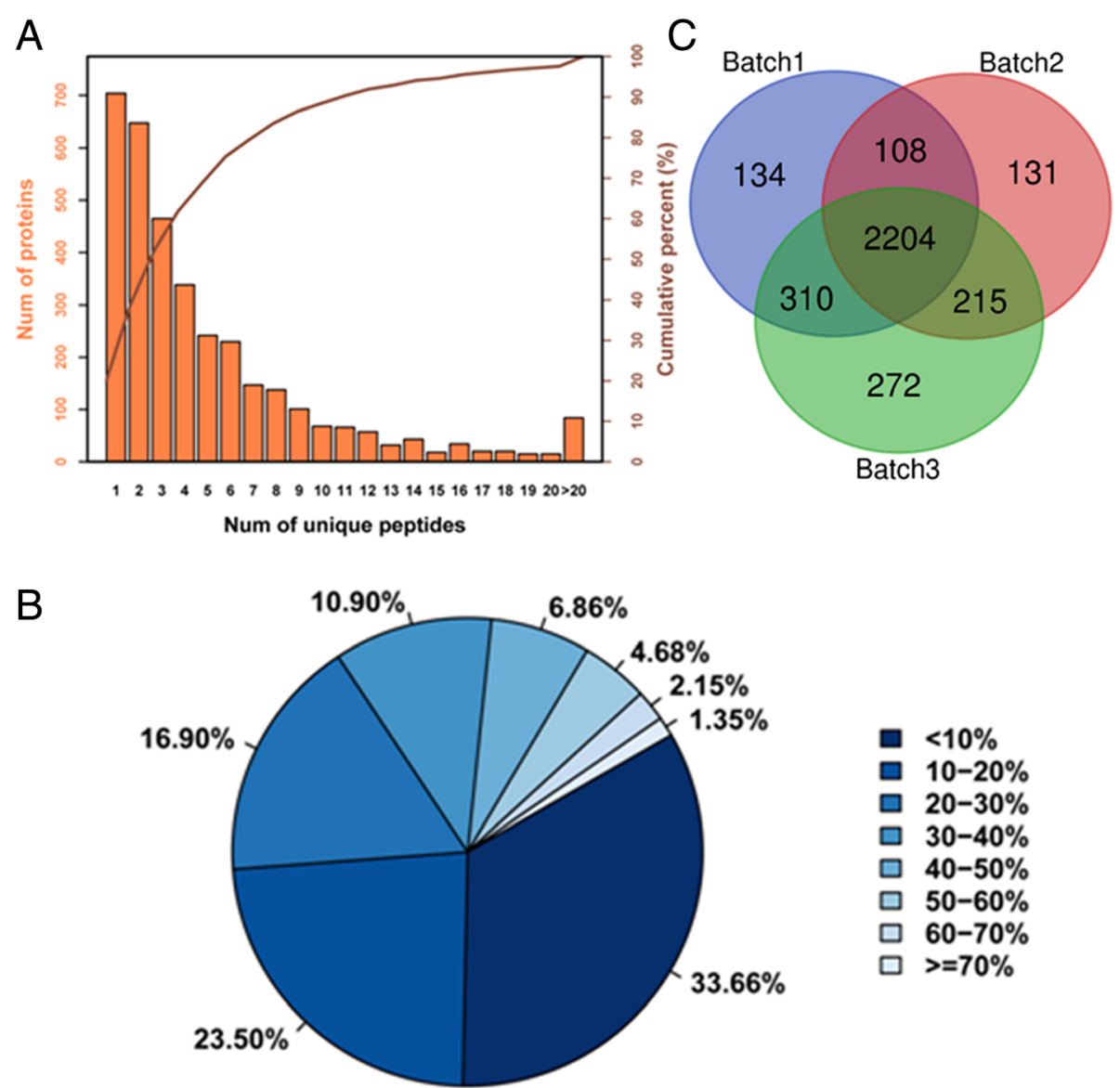

Fig. 3 Identification and analysis of the proteome by iTRAQ a Distribution of the number of peptides. The X axis represents the scope of the number of unique peptides, and the $Y$ axis represents the number of proteins and corresponding cumulative percent. $\mathbf{b}$ Distribution of the proteins'sequence coverage. The pie chart displays the proportion of the number of the different proteins within the scope of coverage in the total protein amount. $\mathbf{c}$ Venn diagram of unique and shared proteins of three batches

Among all three replicates, all proteins were annotated to 52 gene ontology (GO) terms by GO analysis (Additional file 5: Table S2). In terms of biological process categories, most proteins were categorized into the metabolic process $(3193,74.6 \%)$, cellular process $(3166,74.0 \%)$ and response to stimulus $(1364,31.9 \%)$. The major cellular components were cell $(3692,86.2 \%)$ and cell part (3687, $86.1 \%)$. The largest molecular functions of proteins obtained by GO analysis were catalytic activity $(2118,49.5 \%)$ and binding (1998, 46.7\%). Using pathway analysis, 2884 proteins were annotated to 116 pathways including metabolic pathways, Biosynthesis of secondary metabolites, microbial metabolism in diverse environments, ribosome, spliceosome, plant-pathogen interaction, etc.

\section{Identification of DAPs after B. tabaci infestation}

To examine DAPs in response to $B$. tabaci, the proteome changes between two genotypes in response to $B$. tabaci challenge were investigated in three independent iTRAQ experiments. Compared to the control group, a
1.50 -fold or 0.67 -fold change threshold with a $P$-value < 0.05 in protein expression in at least two experiments were classified as a physiologically significant change. We analyzed DAPs between RG inoculated with $B$. tabaci (RB) and RG control (RC), between SG inoculated with B. tabaci (SB) and SG control (SC), and between RC and SC. A total of 115 DAPs was identified from "RB-RC", "SB-SC" and "RC-SC". A venn diagram including the total 115 proteins was generated (Fig. 4). The detailed information of all proteins obtained from three biological replicates is presented in Table 1. After B. tabaci infestation, 24 and 10 DAPs emerged differential accumulation in RB and SB, respectively, but these proteins have no difference in RC-SC. Of the 37 DAPs in $\mathrm{RB}, 18$ proteins were up-regulated and 19 proteins were down-regulated. Among 17 DAPs of SB, 10 proteins were up-regulated and 7 proteins were downregulated. However, 9 and 3 protein levels had changed in RC compared with SC (RC-SC)". These specific $B$. tabaci responsive proteins might be important factor for 


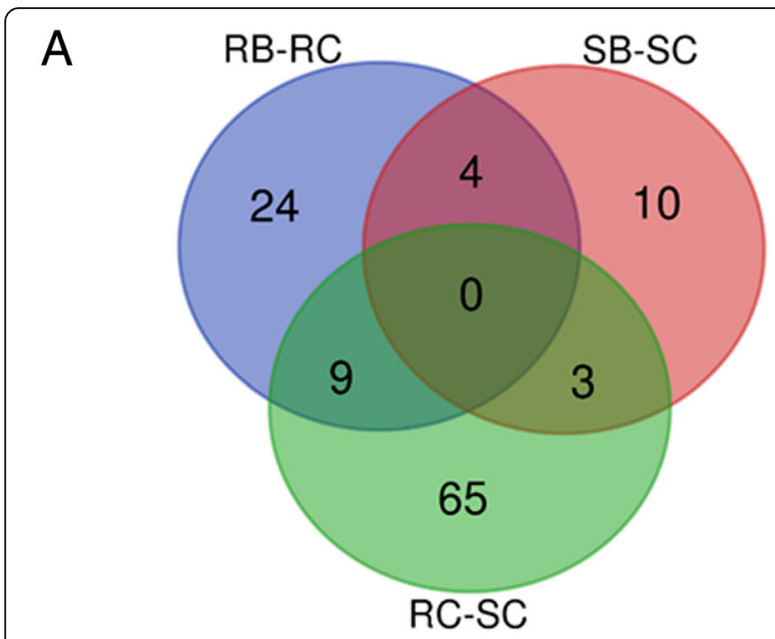

B

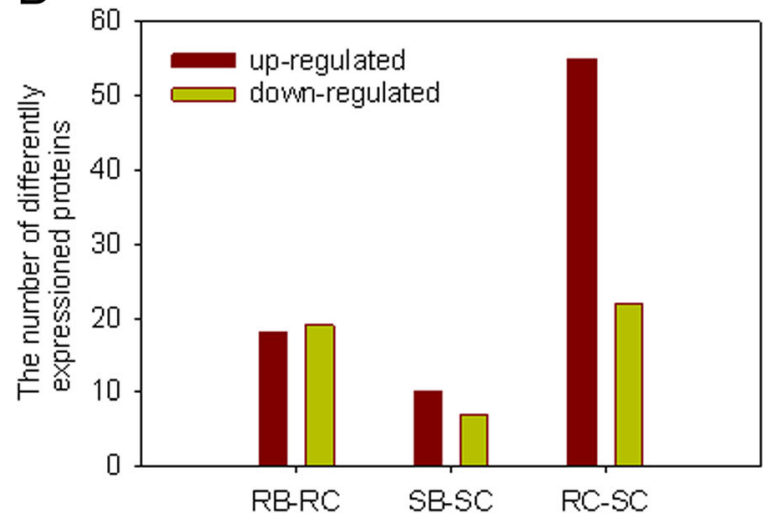

Fig. 4 Venn diagram of differential abundance proteins (DAPs). a Venn diagram; $\mathbf{b}$ The number of up-regulated and down-regulated DAPs

resistance to $B$. tabaci. In the venn diagram, 4 DAPs were shared between two genotypes after $B$. tabaci attack, including $60 \mathrm{~S}$ ribosomal protein L4, xyloglucan endotransglucosylase/hydrolase, acidic endochitinase pcht28-like (PR3) and monodehydroascorbate reductase 5 (MDHAR5).

\section{Classification of DAPs}

On the basis of gene ontology (GO) annotations, the 115 differential abundance proteins (DAPs) were grouped into three major enrichment categories using Blast2GO (Fishers exact test, FDR $<0.05$ ). The GO results showed that 101 DAPs (90.99\%) had been annotated into 37 functional groups, including 15 biological processes, 14 cellular components and 8 molecular function (Fig. 5). In the biological process, the DAPs were mainly involved in metabolic process, cellular process, response to stimulus, and so on. The 'response to stimulus (GO:0051716)', mainly including 'response to oxidative stress (GO:0006979)', existed in all of "RB-RC", "SB-SC" and "RC-SC", corresponding to the process of "hydrogen peroxide metabolic process (GO:
0042743)'. Besides, a higher proportion of up-regulated 'response to stimulus' proteins was existed in "RB-RC" (Additional file 6: Table S3). Furthermore, more photosynthesisrelated protein changes existed in "RC-SC", such as photosynthesis (GO:0015979), plastid organization (GO: 0009657), and light reaction (GO:0019684) (Additional file 6: Table S3). In the molecular function category, the DAPs were mainly involved in catalytic activities and binding (Additional file 7: Table S4). In addition to these two major categories, additional categories identified corresponded to structural molecule activities (GO:0005198), antioxidant activities (GO:0016209), electron carrier activity (GO: 0009055), and so on. A higher proportion of 'hydrolase activity (GO:0004553)' proteins was found in "RB-RC" and "SB-SC" (Additional file 7: Table S4). At the cellular component category, though 'extracellular region' existed in all of "RB-RC", "SB-SC" and "RC-SC", a higher proportion of increased proteins existed in "RB-RC" and "RC-SC", indicating that the related extracellular proteins including cell wall proteins are changed to improve tolerance to $B$. tabaci infestation (Fig. 5; Additional file 8: Table S5). Besides, 'chloroplast' also existed in the three ratio parameters, but the higher proportion of increased proteins in chloroplast existed in "SB-SC" and "RC-SC" (Additional file 8: Table S5), suggesting that photosynthesis-related proteins play an important role in pepper resistance to B. tabaci.

For further functional categorization, KEGG pathway analyses were performed using the KOBAS3.0 database and all DAPs were assigned to 16 KEGG pathways $(P<$ 0.05). All of the proteins in KEGG categories were shown in Additional file 9: Table S6. In "RB-RC", DAPs were assigned to ribosome (ko03010), peroxisome (ko04146), protein processing in endoplasmic reticulum (ko04141), and so on, whereas DAPs were assigned to ascorbate and aldarate metabolism (ko00053) and carbon fixation (ko00710) in "SB-SC". In "RC-SC", it was found that the DAPs participated in the resistant related pathways, such as photosynthesis (ko00195), carbon fixation (ko00710), RNA degradation (ko03018), glycolysis/ gluconeogenesis (ko00010), peroxisome (ko04146) and linoleic acid metabolism (ko00591). Pathways common to "RB-RC", "SB-SC" and "RC-SC" include carbon fixation and linolenic acid metabolism. To gain more insight into photosynthesis-related protein for tolerance to B. tabaci, the DAPs of "RB-RC", "SB-SC" and "RCSC" were analyzed in Additional file 1: Figure S1 and Additional file 2: Figure S2. Based on the GO function and KEGG pathway analysis, multiple proteins involved in stimulus response, antioxidant defense, photosynthesis and linoleic acid metabolism may play defensive role against the B. tabaci damage. A master table (Table 1) that summarized all changed proteins was generated to obtain an overview of the proteins in response to $B$. tabaci. 
Table 1 List of differentially expressed proteins in the resistant and sensitive pepper genotypes infested by B. tabaci

\begin{tabular}{|c|c|c|c|c|c|}
\hline $\begin{array}{l}\text { Biological } \\
\text { function }\end{array}$ & Uniprot ID & Protein name & R_B:R_C & S_B:S_C & R_C:S_C \\
\hline \multicolumn{6}{|c|}{ Redox regulation } \\
\hline & A0A1U8ESV4 & Catalase (CAT2) & 8.69 & 1.84 & 0.23 \\
\hline & A0A1U8FQG1 & Peroxidase (POD) & 6.36 & 1.60 & 0.43 \\
\hline & A0A1U8EZN6 & Heme-binding protein 2-like (HBP1) & 3.02 & 0.57 & 5.41 \\
\hline & A0A1U8FBV9 & Putative quinone-oxidoreductase homolog (CEQORH) & 2.87 & 1.56 & 0.39 \\
\hline & A0A1U8EZE6 & Heme-binding protein 2-like (HBP1) & 2.69 & 0.89 & 18.52 \\
\hline & A0A1U8FMA2 & Catalase (CAT) & 1.98 & 1.27 & 2.40 \\
\hline & A0A1U8GY32 & Glutathione S-transferase (GST) & 1.87 & 1.36 & 1.31 \\
\hline & A0A1U8GBS1 & Glutathione reductase (GR) & 1.52 & 0.73 & 2.89 \\
\hline & A0A089FZ95 & Dehydroascorbate reductase (DHAR) & 1.30 & 0.79 & 2.79 \\
\hline & A0A1U8HAQ7 & Uncharacterized oxidoreductase & 1.29 & 0.78 & 2.01 \\
\hline & A0A1U8EL92 & 2-methylene-furan-3-one reductase (AOR) & 1.22 & 0.69 & 1.96 \\
\hline & A0A1U8E8C1 & Peroxiredoxin-2E-2(PRXIIE) & 1.07 & 2.40 & 1.04 \\
\hline & A0A1U8F1N7 & Peroxisomal (S)-2-hydroxy-acid oxidase (GLO1) & 0.99 & 1.10 & 2.00 \\
\hline & A0A1U8E6R6 & Monodehydroascorbate reductase (MDHAR) & 0.85 & 1.86 & 1.08 \\
\hline & A0A1U8GEC0 & Monodehydroascorbate reductase 5 (MDHAR5) & 0.41 & 1.91 & 1.40 \\
\hline & A0A1U8H5G9 & Superoxide dismutase (SOD) & 0.27 & 1.59 & 4.27 \\
\hline \multicolumn{6}{|c|}{ Response to stress } \\
\hline & A0A1U8FME6 & Acidic endochitinase pcht28-like (PR3) & 32.37 & 14.23 & 0.02 \\
\hline & B2CZJ6 & PR10 & 19.57 & 1.74 & 0.08 \\
\hline & A0A1U8FJE1 & Probable carboxylesterase (CXE6) & 10.83 & 4.61 & 2.70 \\
\hline & A0A1U8E530 & Annexin D4-like (ANN4) & 7.63 & 1.61 & 0.64 \\
\hline & A0A1U8H0C7 & Calreticulin-3 (CRT3) & 3.72 & 1.37 & 0.73 \\
\hline & A0A1U8HDQ1 & Flower-specific defensin-like & 3.64 & 0.71 & 0.92 \\
\hline & A0A1U8FWW2 & Heat shock 70 kDa protein (HSP70.1) & 2.74 & 1.28 & 0.83 \\
\hline & A0A1U8EMR4 & Glutamate--glyoxylate aminotransferase 2 (AOAT2) & 2.50 & 1.17 & 1.12 \\
\hline & A0A1U8ELM1 & Heat shock protein 90.5 (HSP90) & 1.38 & 0.77 & 1.90 \\
\hline & A0A1U8GCN1 & Pathogenesis-related protein STH-2-like (NUP98B) & 1.38 & 4.33 & 0.19 \\
\hline & A0A1U8FJF5 & CSC1-like protein ERD4(Early-responsive to dehydration stress protein (ERD4) & 1.20 & 0.76 & 1.86 \\
\hline & A0A1U8EXS4 & Putative amidase C869.01 (AmidP) & 1.13 & 1.50 & 0.28 \\
\hline & A0A1U8E2L2 & Cysteine-rich repeat secretory protein 38-like (CRRSP38) & 1.05 & 1.26 & 0.32 \\
\hline & A0A1U8E6Q9 & Stromal 70 kDa heat shock-related protein (CPHsp70.2) & 0.97 & 0.87 & 2.12 \\
\hline & A0A1U8GL40 & Stress protein DDR48-like & 0.89 & 0.57 & 0.24 \\
\hline & A0A1U8EX11 & Patatin & 0.87 & 1.25 & 12.23 \\
\hline & A0A1U8GH17 & Kirola-like & 0.64 & 0.17 & 0.64 \\
\hline & A0A1U8G6G8 & Chitin-binding lectin 1-like & 0.54 & 1.12 & 0.35 \\
\hline & A0A1U8EN72 & plasma membrane-associated cation-binding protein 1 & 0.51 & 0.71 & 1.18 \\
\hline & E9JEC2 & Mannose-binding lectin OS=Capsicum annuum & 0.46 & 1.76 & 0.21 \\
\hline & Q42493 & Fibrillin & 0.45 & 2.09 & 2.89 \\
\hline & A0A1U8EKU6 & Myristoylated alanine-rich C-kinase substrate-like & 0.41 & 1.42 & 1.55 \\
\hline & A0A1U8EZY1 & Stromal $70 \mathrm{kDa}$ heat shock-related protein & 0.31 & 1.21 & 1.21 \\
\hline \multicolumn{6}{|c|}{ Protein metabalism and Regulation } \\
\hline & A0A1U8GAJ4 & Endoplasmin homolog & 2.69 & 1.51 & 0.89 \\
\hline
\end{tabular}


Table 1 List of differentially expressed proteins in the resistant and sensitive pepper genotypes infested by B. tabaci (Continued)

\begin{tabular}{|c|c|c|}
\hline $\begin{array}{l}\text { Biological } \\
\text { function }\end{array}$ & Uniprot ID & Protein name \\
\hline & A0A1U8G5J6 & Chaperone protein ClpB1-like (HSP101) \\
\hline & A0A1U8GVQ2 & Protein disulfide-isomerase (PDIL6) \\
\hline & A0A1U8E845 & Diaminopimelate epimerase (DAPE) \\
\hline & A0A1U8FD80 & Aspartyl protease AED3 \\
\hline & A0A1U8GG27 & Probable serine protease EDA2 \\
\hline & A0A1U8E900 & 20 kDa chaperonin, chloroplastic-like (CPN20) \\
\hline & A0A1U8GX36 & Protein disulfide-isomerase (PDIL1) \\
\hline & A0A1U8DX55 & Presequence protease 1 (PreP2) \\
\hline & A0A1U8FITO & Uncharacterized protein \\
\hline & A0A1U8E5E9 & $60 S$ ribosomal protein $L 4$ \\
\hline & A0A1U8GMG9 & $60 S$ ribosomal protein $\mathrm{L} 13$ \\
\hline & J7HAU1 & $50 S$ ribosomal protein $L 2$ \\
\hline & A0A1U8DRN2 & $50 S$ ribosomal protein $L 3$ \\
\hline & A0A1U8HJ31 & 30 S ribosomal protein $\mathbf{S} 20$ \\
\hline & A0A1U8GWX0 & Uncharacterized protein \\
\hline & A0A1U8HNK6 & 605 ribosomal protein L7a-1 \\
\hline & A0A1U8ELW8 & $50 S$ ribosomal protein L15 \\
\hline \multicolumn{3}{|c|}{ Lipid related metabolism } \\
\hline & A0A1U8E9J9 & Peroxisomal fatty acid beta-oxidation multifunctional protein AIM1-like \\
\hline & A0A1U8EK22 & Probable plastid-lipid-associated protein 13 \\
\hline & F2YL87 & Lipoxygenase (LOX2) \\
\hline & A0A1U8FRJ4 & Uncharacterized protein (PLDRP1) \\
\hline & A0A1U8F9H1 & Phospholipase D (PLD) \\
\hline & A0A1U8EAJ0 & Patellin-3-like \\
\hline
\end{tabular}

Phototsynthesis related proteins

\begin{tabular}{|c|c|c|c|c|}
\hline A0A1U8E7W8 & Malic enzyme (ME1) & 4.37 & 1.41 & 0.36 \\
\hline A0A1U8FJN4 & Oxygen-evolving enhancer protein 1 & 2.36 & 1.06 & 4.79 \\
\hline A0A1U8GUM8 & ATP synthase subunit b & 2.19 & 0.75 & 3.88 \\
\hline A0A1U8ESR9 & Serine--glyoxylate aminotransferase (AGT1) & 2.09 & 1.01 & 1.01 \\
\hline A0A1U8FZN5 & Oxygen-evolving enhancer protein 1 & 1.96 & 1.19 & 2.34 \\
\hline A0A1U8FYP5 & Carbonic anhydrase (CAT) & 1.90 & 2.10 & 2.26 \\
\hline A0A1U8FGM0 & Photosystem II repair protein PSB27-H1 & 1.89 & 0.63 & 2.45 \\
\hline $\mathrm{A} 0 \mathrm{~A} 1 \mathrm{U} 8 \mathrm{E} 7 \mathrm{H} 4$ & Ribulose bisphosphate carboxylase/oxygenase activase 1 & 1.86 & 1.16 & 3.22 \\
\hline A0A1U8HDS6 & Phosphoglycerate kinase (PGK2) & 1.81 & 1.36 & 1.92 \\
\hline A0A1U8GDS4 & Fructose-1,6-bisphosphatase & 1.75 & 1.07 & 3.03 \\
\hline A0A1U8FUM0 & ATP synthase gamma chain & 1.71 & 0.99 & 2.90 \\
\hline A0A1U8FMQ7 & Ferredoxin--NADP reductase & 1.61 & 0.74 & 2.99 \\
\hline A0A1U8FRH4 & Cytochrome f-like & 1.43 & 0.97 & 2.83 \\
\hline A0A1U8FHQ4 & Fructose-bisphosphate aldolase & 1.41 & 1.07 & 2.31 \\
\hline A0A1U8FNB3 & Transketolase (TKL1) & 1.39 & 0.95 & 2.41 \\
\hline A0A1U8E6P3 & Enolase & 1.32 & 0.85 & 1.77 \\
\hline A0A1U8GVK4 & Photosystem I reaction center subunit II & 1.24 & 0.95 & 2.00 \\
\hline A0A1U8HK56 & LOW QUALITY PROTEIN: phosphoenolpyruvate carboxylase-like (PPC2) & 1.23 & 2.62 & 2.31 \\
\hline
\end{tabular}


Table 1 List of differentially expressed proteins in the resistant and sensitive pepper genotypes infested by B. tabaci (Continued)

\begin{tabular}{|c|c|c|c|c|c|}
\hline $\begin{array}{l}\text { Biological } \\
\text { function }\end{array}$ & Uniprot ID & Protein name & R_B:R_C & S_B:S_C & R_C:S_C \\
\hline & A0A1U8HFF2 & RuBisCO large subunit-binding protein subunit beta & 1.16 & 0.74 & 2.65 \\
\hline & A0A1U8FQ68 & LOW QUALITY PROTEIN: photosystem II stability/assembly factor & 1.14 & 0.91 & 1.84 \\
\hline & A0A1U8GZ15 & Sedoheptulose-1,7-bisphosphatase & 1.09 & 0.90 & 2.20 \\
\hline & K4FWQ6 & Citrate synthase (CS) & 1.06 & 1.77 & 0.42 \\
\hline & J7H3N5 & Photosystem II protein D1 & 1.04 & 0.51 & 0.59 \\
\hline & A0A1U8EAE0 & Oxygen-evolving enhancer protein 2 & 0.97 & 0.76 & 2.48 \\
\hline & A0A1U8HDT5 & Porphobilinogen deaminase & 0.94 & 0.79 & 2.25 \\
\hline & A0A1U8H8P7 & NADP-dependent glyceraldehyde-3-phosphate dehydrogenase & 0.94 & 1.94 & 0.30 \\
\hline & A0A1U8GAG1 & ATP-dependent zinc metalloprotease FTSH & 0.86 & 0.77 & 1.66 \\
\hline & O78327 & Transketolase 1 (TKL1) & 0.84 & 3.24 & 2.69 \\
\hline & A0A1U8EJC2 & RuBisCO large subunit-binding protein subunit alpha & 0.84 & 0.81 & 2.80 \\
\hline & A0A1U8EC90 & magnesium-protoporphyrin IX monomethyl ester [oxidative] cyclase & 0.64 & 0.68 & 1.39 \\
\hline \multicolumn{6}{|c|}{ Carbon metabolism related proteins } \\
\hline & A0A1U8HHZ9 & Benzyl alcohol O-benzoyltransferase & 5.54 & 0.72 & 0.54 \\
\hline & A0A1U8GAR2 & Cinnamoyl-CoA reductase & 2.46 & 1.08 & 2.54 \\
\hline & A0A1U8FQ55 & Serine hydroxymethyltransferase (SHMT) & 1.22 & 0.99 & 1.80 \\
\hline & A0A1U8E7Q2 & Soluble inorganic pyrophosphatase 6 (PPA6) & 1.01 & 0.87 & 3.05 \\
\hline & A0A1U8F9K2 & Probable Xaa-Pro aminopeptidase P & 0.90 & 1.12 & 1.89 \\
\hline & A0A1U8HKG3 & Glucose-1-phosphate adenylyltransferase (APL1) & 0.84 & 0.73 & 2.31 \\
\hline & A0A1U8EIA9 & Alpha-L-arabinofuranosidase & 0.83 & 1.05 & 0.44 \\
\hline & A0A1U8F8D2 & Pectinesterase & 0.82 & 1.32 & 0.22 \\
\hline & A0A1U8DZQ7 & Glucose-1-phosphate adenylyltransferase (APL1) & 0.81 & 0.86 & 2.06 \\
\hline & A0A1U8DSA1 & Uncharacterized protein & 0.61 & 0.22 & 0.37 \\
\hline & A0A1U8F6S1 & 5-methyltetrahydropteroyltriglutamate-homocysteine methyltransferase & 0.51 & 0.82 & 0.74 \\
\hline & A0A1U8FQ91 & Xyloglucan endotransglucosylase/hydrolase (XTHs) & 0.37 & 0.22 & 0.62 \\
\hline \multicolumn{6}{|c|}{ Other aspects } \\
\hline & A0A1U8E2F0 & DEAD-box ATP-dependent RNA helicase 3 & 0.56 & 0.83 & 1.07 \\
\hline & A0A1U8EZ49 & DNA-damage-repair/toleration protein (DRT100) & 1.01 & 0.29 & 0.46 \\
\hline & A0A1U8H3L5 & Lysine--tRNA ligase & 1.16 & 1.29 & 0.40 \\
\hline & A0A1U8H5ZO & Protein plastid transcriptionally active 16 & 1.15 & 0.90 & 2.27 \\
\hline & A0A1U8H847 & Extracellular ribonuclease LE-like (RNS3) & 2.53 & 1.00 & 0.06 \\
\hline & A0A1U8H8A4 & Ribonuclease $\mathrm{T} 2$ family protein & 0.97 & 2.26 & 0.03 \\
\hline & A0A1U8HET6 & Ribonuclease S-4-like & 0.96 & 0.94 & 0.21 \\
\hline & A0A1U8EW99 & Protein SIEVE ELEMENT OCCLUSION:protein SIEVE ELEMENT OCCLUSION B-like (SEOR1) & 0.97 & 1.68 & 0.43 \\
\hline & A0A1U8DW72 & Protein EXORDIUM-like 2 (EXL2) & 2.10 & 0.82 & 3.35 \\
\hline & A0A1U8HCV1 & Elongation factor Tu (EF-Tu) & 0.95 & 0.77 & 2.36 \\
\hline
\end{tabular}

Bold indicates proteins considered as being differentially expressed at level of $p$ value of $\leq 0.05$

\section{Validation of iTRAQ data for selected proteins by PRM}

PRM is a recent development in targeted mass spectrometry, which is more specific and sensitive than selected reaction monitoring and has been widely used to quantify and detect target proteins $[15,16]$. In this study, the protein expression levels obtained by iTRAQ were confirmed by quantifying the expression levels of some proteins by PRM-MS analysis. Ten candidate proteins related to resistance to $B$. tabaci were selected for PRM analysis. Among 10 target proteins, 8 proteins have MS/ MS spectrum(s) and unique peptide(s). Therefore, the PRM detection was performed for the 8 protein only (CAT, SOD, PLD, HBP1, LOX2, TKL1, PPA6, APL1). In general, the trends in the change of the results measured 


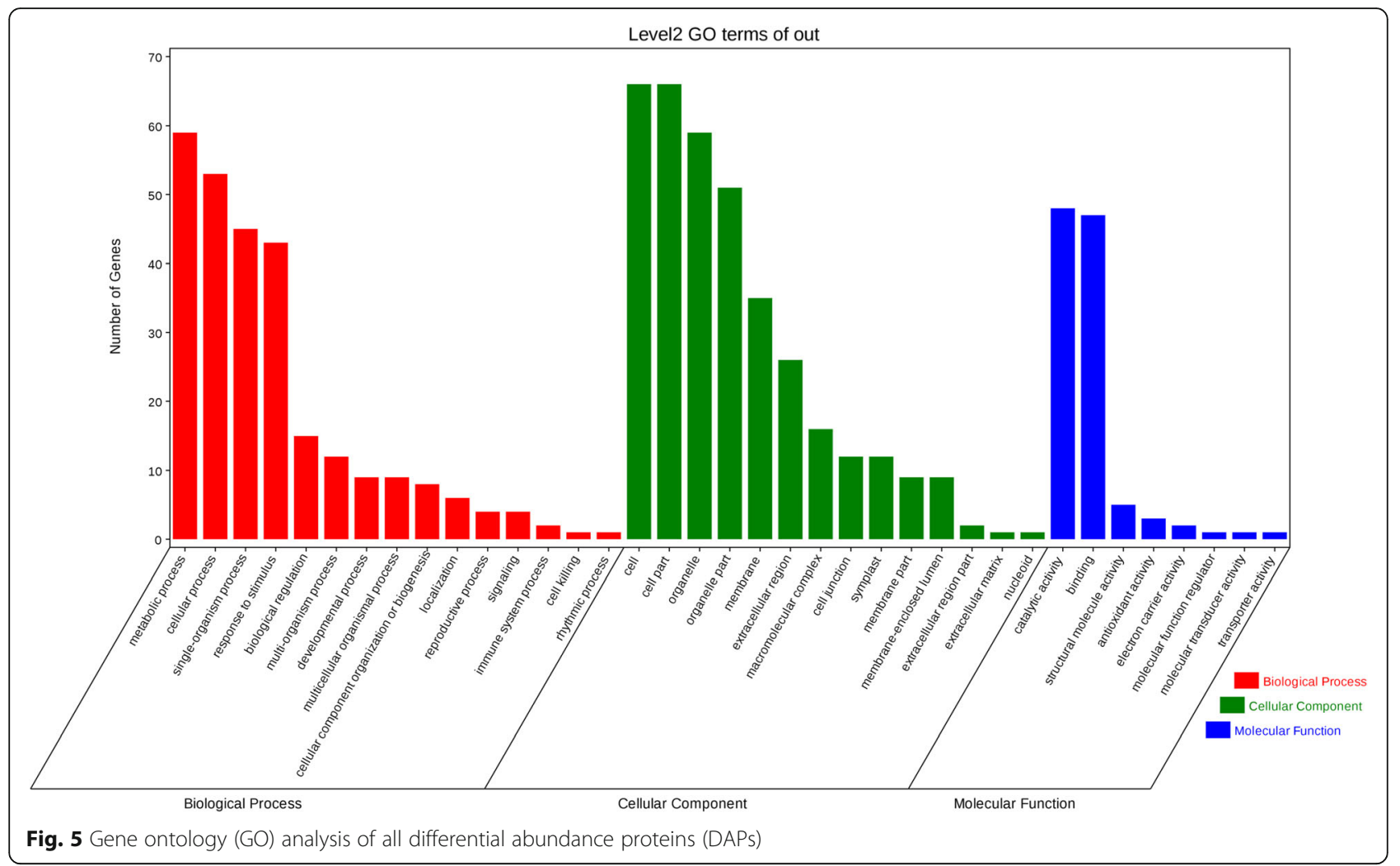

by PRM and iTRAQ are basically consistent (Fig. 6). However, there is difference between the actual value. The difference between the values may be due to the different detection methods $[16,17]$. Therefore, our iTRAQ results are reliable and reproducible.

\section{Confirmation of iTRAQ data for selected proteins by qRT- PCR}

To further confirm the iTRAQ data, we monitored the expression patterns of the corresponding genes encoding proteins using qRT-PCR. The expression patterns of the six genes (CAT, SOD, PLD, HBP1, LOX2, PR3) are shown in Fig. 7. The expression trends of four genes basically matched with our iTRAQ data, except for the other two genes (CAT and HBP1). The low correlation coefficient of the proteome and transcriptome data has been reported previously $[18,19]$. The discrepancies could potentially be attributed to mRNA stability, splicing, translational regulation, post-translational processing, control of protein turnover, protein degradation or a combination of these [20].

\section{Discussion}

Generation of a comprehensive proteome map of $B$. tabaci infested pepper

$B$. tabaci is a major pest of both greenhouse and openfield horticultural crops. Screen and utilization of resistant plants to control B. tabaci is an important mean of agricultural production. To date, our understanding of the molecular mechanism underlying the defense response of plants to B. tabaci is limited, particularly of resistant plants. Proteomics has emerged as a powerful tool to explore physiological changes at the cellular level, but few attempts have been made to study the response of pepper to B. tabaci attack at the level of proteome. Yin et al. [6] showed the proteome change of $A$. thaliana leaves infested by B. tabaci using two-dimensional electrophoresis. In that report, however, a single Arabidopsis cultivar was used for B. tabaci infestation and only 20 proteins were generated. In the present study, the distinct genetic background of RG and SG genotypes provided a solid foundation for identifying proteins involved in the pepper defense response against B. tabaci attack. To our knowledge, it is the first time that the advanced proteomic technology (such as iTRAQ) was used to study B. tabaci resistant mechanisms using two different resistant materials.

Two methods were utilized to fully assess the iTRAQ differential expression data (Additional file 10: Table S7). For the first one, a 1.50 -fold or 0.67 -fold change threshold with a $P$-value $<0.05$ in the average value of three replicates was classified as a physiologically significant change. A total 398 DAPs were identified in the three classes of differentially accumulated proteins of "RB-RC" (130), "SB-SC" (139) and "RC-SC" (251). To further search for key protein components or pathways for $B$. 

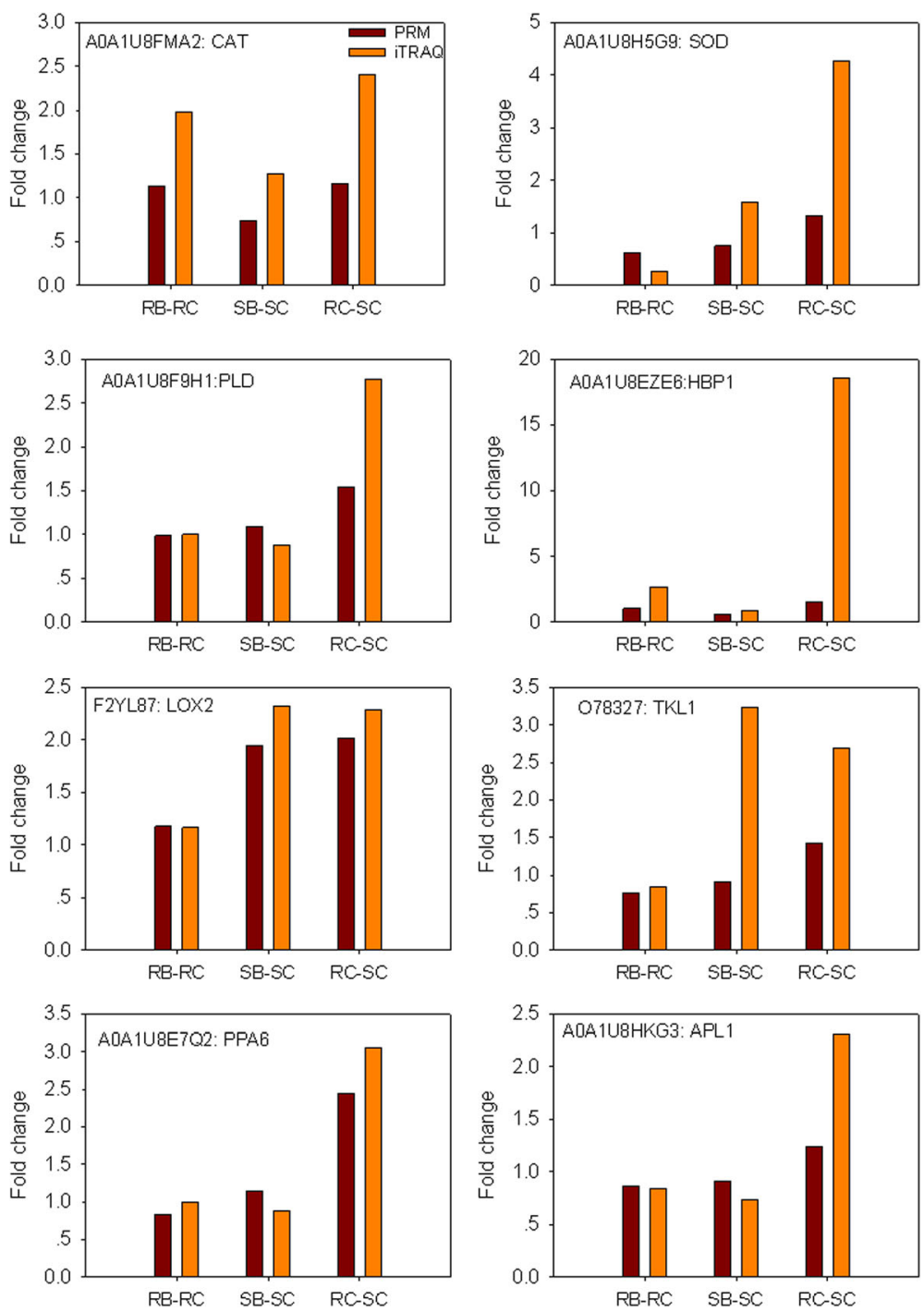

Fig. 6 Relative expression levels of selected proteins measured by PRM in the RB-RC, SB-SC and RC-SC. RB-RC represents protein level changes in the resistant genotype after $B$. tabaci infestation; SB-SC represents protein level changes in the susceptible genotype after B. tabaci infestation; RC$\mathrm{SC}$ represents protein level changes in the resistant/susceptible genotype under control conditions. The protein samples for PRM were exacted from peppers treated with B. tabaci for $48 \mathrm{~h}$

tabaci resistance response, a 1.50 -fold or 0.67 -fold change threshold with a $P$-value $<0.05$ in at least two experiments and in the average value of three replicates were classified as a significant change. A total 115 DAPs were identified in the three classes of differentially accumulated proteins of "RB-RC" (37), "SB-SC" (17) and "RC-SC" (77). More DAPs were present in the $\mathrm{RB}$ than $\mathrm{SB}$. The results are supported by previous studies reporting that $B$. tabaci infestations drive more differentially expressed genes (DEGs) in the strong resistance cultivar than sensitivity [13] Besides, differential expression analyses revealed that more upregulated proteins were identified than downregulated in the classes of "SB-SC" and "RC-SC".
However, our results contrast with that observed in cotton stressed by B. tabaci, in which downregulation > up-regulation of DEGs. The inconsistence in proteins and genes may explain the discrepancy in the translational and post-translational regulations in pepper defense against $B$. tabaci infection. In the present study, the function of DAP from the second method was further analyzed and discussed in the following sections. GO enrichment and KEGG pathway analysis indicated that proteins involved in processes such as oxidative stress regulation, stimulus response, linoleic acid metabolism and photosynthesis might be involved in the host plant resistance to B. tabaci infestation. 

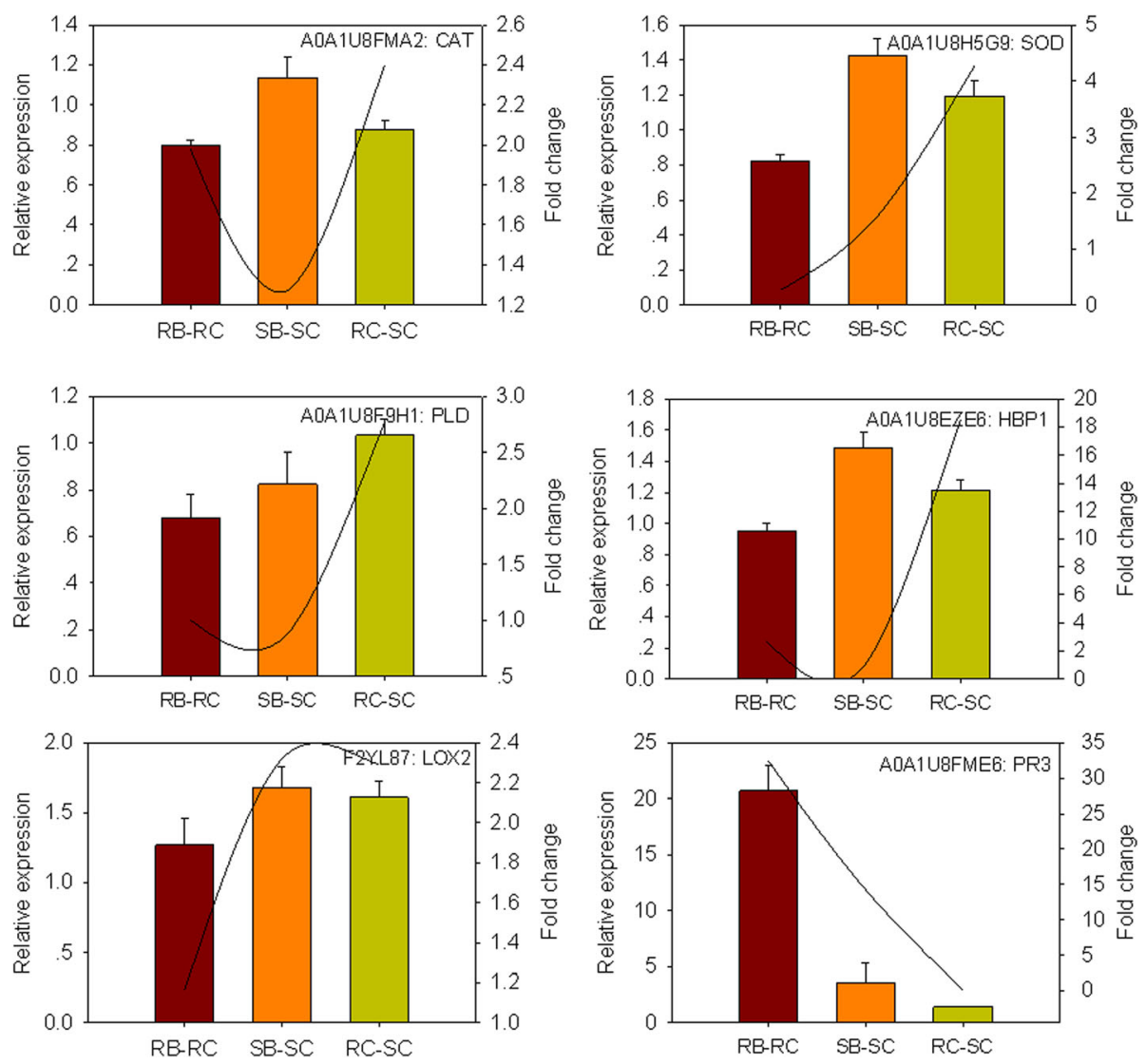

Fig. 7 Real-time PCR analysis of genes encoding the selected proteins in RB-RC, SB-SC and RC-SC. RB-RC represents protein level changes in the resistant genotype after $B$. tabaci infestation; SB-SC represents protein level changes in the susceptible genotype after $B$. tabaci infestation; RC-SC represents protein level changes in the resistant/susceptible genotype under control conditions. The expression levels of CAT, SOD, HBP1, LOX2, $P L D$ and PR3 were quantified relative to the value obtained from control samples (B. tabaci-free plants). The column means the relative expression level of genes and the line means the trend value of iTRAQ

\section{DAPs involved in oxidative stress}

Reactive oxygen species (ROS) are generated in plant tissues in response to different stresses including the interaction with herbivores [21]. ROS are important signal molecules in plants, but can also cause plant toxicity. Plants have formed a set of enzymatic systems to scavenge highly ROS. In this study, several oxidative stressrelated proteins such as catalase (CAT, A0A1U8FMA2), peroxidase (POD, A0A1U8FQG1), superoxide dismutase (SOD, A0A1U8H5G9), dehydroascorbate reductase (DHAR, A0A089FZ95), glutathione reductase (GR, A0A1U8GBS1) and monodehydroascorbate reductase 5 (MDHAR5, A0A1U8GEC0) were identified in response to $B$. tabaci attack (Table 1).

SOD is the first enzyme with free radicals as the substrate, catalyzing the dismutation of superoxide radicals to $\mathrm{O}_{2}$ and $\mathrm{H}_{2} \mathrm{O}_{2}$, maintaining adequately low oxyradical levels. It is an important protective enzyme in plants' cell defense systems, and is closely related to the plants' resistances [17]. In this study, the SOD was up-regulated in $\mathrm{RC}$ when compared to $\mathrm{SC}$, indicating that the higher resistance materials may display high ability to scavenge ROS. CAT catalyzes decomposed $\mathrm{H}_{2} \mathrm{O}_{2}$ to water and oxygen and CAT are characterized by the oxidation of various organic compounds, which have been shown to be involved in insect response in rice [20]. PODs are one kind of essential enzymes of the immediate response of plants to insect damage. The role of PODs in plant resistance to insect pests has been studied in various plant systems. For example, production of phenoxy and other oxidative radicals by PODs in association with phenols directly deters the feeding by insects and/or produces toxins that reduce the plant digestibility, which in turn leads to nutrient deficiency in insects with drastic effects on their growth [22]. In this study, the iTRAQ data showed that the expression levels of CAT and POD were induced in both pepper genotypes, but were only significantly induced in the RB. It is speculated that both proteins in the resistant pepper have the ability to reduce the ROS damage caused by B. tabaci. MDHAR, DAR and GR are the key enzyme in the ascorbate acid- glutathione (AsA-GSH) cycle [23]. AsA-GSH cycle is 
considered to be an important mechanism for resistance of plants under stress conditions [24]. In this cycle, ascorbate peroxidase (APX) catalyzes the reduction of $\mathrm{H}_{2} \mathrm{O}_{2}$ into water with AsA serving as an electronic donor. DHAR utilizes the electrons provided by GSH to reduce DHA, while DHA is previously produced from MDHA. Simultaneously, GSH is oxidized into glutathione disulfide (GSSG) by DHAR and GSSG is then reduced into GSH, catalyzed by GR. Greenbug feeding on resistant sorghum induced the expression of peroxidase and glutathione-S-transferase genes, but both up- and down-regulated different CAT genes [25].

Arabidopsis MDARP and MDAR3 were also upregulated by $B$. tabaci, which may function to scavenge excessive ROS that result from B. tabaci feeding [6]. Here, three differentially expressed enzymes MDAHR, DAR and GR were detected, which were all up-regulated in $\mathrm{RC}$ when compared to $\mathrm{SC}$, indicating that the high level of AsA-GSH cycle plays an important role in the protection of pepper seedlings against $B$. tabaci injury. Thompson and Goggin [26] showed that phloem-feeding insects (PFIs) did not uniformly regulate the oxidative stress-related genes in whole leaf tissues. Our results also showed that the B. tabaci triggered a differential modulation of antioxidant proteins in both genotypes. For example, B. tabaci infestation up-regulated the expression of CAT and POD, but down-regulated the expression of SOD and MDAHR in the resistant pepper.

Besides, under oxidative stress conditions the lipid constituents of cells can undergo oxidation, which is toxic to biomolecules and several enzyme activities. Recently, CeQORH was reported to reduce the double bond of stress-related oxidized lipids named $\gamma$-ketols [27]. In the present study, A0A1U8FBV9 (CeQORH) is a chloroplast envelope quinone oxidoreductase homolog, which was induced by B. tabaci in the SC (Table 1). Haem is prominent among the iron binding molecules in the cell. The presence of free haem in the cytoplasm must be maintained at a low concentration to prevent oxidative stress through the oxidation of haem iron [28]. A0A1U8EZE6 and A0A1U8EZN6 (HBP) are hemebinding protein-like protein, which were induced by $B$. tabaci in the RB, but not in SB, and had a higher level in the $\mathrm{RC}$ compared to the SC. Early researches have confirmed that Arabidopsis homologous gene AtHBP5 was involved in antioxidant pathway. AtHBP5 overexpressing plants show a decreased accumulation of $\mathrm{H}_{2} \mathrm{O}_{2}$. It is proposed that the interaction between the HY1 and AtHBP5 proteins participate in an antioxidant pathway that might be mediated by reaction products of haem catabolism [29]. Oxidative stress is one of the first general reactions to the injuries caused by insects when they penetrate the plant [20]. Our results suggest that the resistant pepper has a high ability to cope with the oxidative damage and B. tabaci-triggered differential regulation of antioxidant proteins is possibly due to different degrees of disruption of cell redox homeostasis.

\section{DAPs involved in stress response}

Plants are endowed with constitutive and inducible protective mechanisms of biotic-/abiotic-defense known as the stress or defense responses [30,31]. In the present study, several stress-related proteins were differentially regulated in both genotypes, among which, three proteins are HSP type (A0A1U8E6Q9, A0A1U8ELM1, A0A1U8FVW2). A0A1U8E6Q9 is a stromal $70 \mathrm{kDa}$ heat shock-related protein and its homologous protein, Arabidopsis CPHsp70.2, is required for protection against oxidative stress in Arabidopsis thaliana [32]. A0A1U8ELM1 is a heat shock protein 90.5, which is a chloroplast localized HSP90 family molecular chaperone in Arabidopsis, and it has been implicated in plant abiotic stress resistance, photomorphogenesis and nuclear-encoded protein import into the chloroplast [33]. A0A1U8FVW2 is a heat shock $70 \mathrm{kDa}$ protein (Hsp70-1), and cytosolic Hsp70s have been shown to be involved in the thermotolerance and the immune response in Arabidopsis, and be required for productive potyvirus infection of tobacco plants [34]. From our iTRAQ data, CPHsp70.2 and Hsp90.5 showed significant accumulation (about 2fold) in RC-SC, but Hsp70-1 was only upregulated in the $\mathrm{RB}$. The change in the expression profile indicates that these HSPs are involved in B. tabaci tolerance.

Pathogenesis-related proteins (PR proteins) and defense-related proteins are specifically induced under stress conditions [31]. Three biotic stress-related proteins were identified as PR protein (A0A1U8FME6, A0A1U8HDQ1, B2CZJ6), of which the expression level in the $\mathrm{RB}$ were higher than that in the $\mathrm{SB}$ (Table 1). A0A1U8FME6 is one kind of PR3 protein, encoding a basic chitinase involved in ethylene/jasmonic acid mediated signaling pathway during systemic acquired resistance. PR3 genes were induced systemically but not locally and they can be candidates for broad-spectrum resistance, viz., induced systemic resistance [35]. A0A1U8HDQ1 encodes a member of a family of small, secreted, cysteine rich protein with sequence similarity to the PCP (pollen coat protein) gene family. B2CZJ6 encodes a member of the PYR (pyrabactin resistance)/ RCAR5 (regulatory components of ABA receptor) family proteins, which is also known as PR10 [36]. Overexpression of RCAR5 resulted in ABA-hypersensitive phenotypes and enhanced the resistance of Arabidopsis plants to Pseudomonas syringae pv. tomato (Pst) DC3000, through promoting stomata closure leading to the development of resistance to this bacterial pathogen [37]. PR proteins not only inhibit pathogen progress but also help in host plants growth, which accumulate locally in the infected and surrounding tissues. Production of PR 
proteins in the uninfected plant's parts can prevent the affected plants from further infection [35]. These results suggest that these PRs may be involved in plant perception and responses to $B$. tabaci attack signals.

Other stress-related proteins like annexin D4-like (ANN4, A0A1U8E530) and calreticulin-3 (CRT3, A0A1U8H0C7) were increased in the leaves when the $B$. tabaci were feeding on the pepper, of which the expression was significantly increased in the RB than that in the SB. ANN4 encodes $\mathrm{Ca}^{2+}$-regulated membranebinding proteins modulating cytosolic calcium signatures. Huh et al. [38] provided experimental evidence that AnnAt4 and AnnAt1 interact with each other in a $\mathrm{Ca}^{2+}$-dependent manner and function to regulate responses to drought and salt stress. Recently, ANN4mediated cytosolic calcium signaling was reported to be involved in MYB30-regulating oxidative and heat stress responses in Arabidopsis [39]. In our experiment, ANN4 expression was significantly induced in the $R B$, reaching 7.63-fold, but remained unchanged in the SB, indicating that ANN4 and ANN4-mediated calcium signaling may be involved in pepper resistant to $B$. tabaci damage. CRT has been documented to be a $\mathrm{Ca}^{2+}$-binding molecular chaperone that facilitates the folding of newly synthesized glycoproteins and regulates the $\mathrm{Ca}^{2+}$ homeostasis in the endoplasmic reticulum (ER) lumen [40]. It has been suggested that Arabidopsis CRT3 mediate plant defense against viral and biotrophic pathogens [41] Arabidopsis CRT mutant atcrt3 is more sensitive Pseudomonas syringae pv tomato DC3000 (Pst DC3000) and water stress $[40,41]$. Therefore, a high expression of CRT3 in the highly resistant pepper may be determined to be a defense against $B$. tabaci invasion.

\section{DAPs involved in protein metabolism and regulation}

When plants are attacked by insects, they produce many defense-related proteins, many of which are synthesized and then secreted to their various destinations within the cell [20]. In terms of protein synthesis, six ribosomal proteins such as $30 \mathrm{~S}$ ribosomal protein S20, 50 S ribosomal protein L2/L15 and 60S ribosomal protein L4/L13/ $7 \mathrm{a}-1$, showed higher abundance in the $\mathrm{RC}$ than in the SC. However, these ribosomal proteins were downregulated in the RB, but remained unchanged in the SB. The results indicate that protein synthesis processes in resistant plants can maintain a high level, although the exact influencing mechanism is still unclear.

Protein disulfide isomerase (PDI) is a member of the thioredoxin superfamily, and is involved in the progression and maturation of secretory proteins in the ER [42, 43]. AtPDI6 acts as an attenuator of D1 synthesis, modulating photo inhibition in a light-regulated manner. PDI is a component of unfolded protein response that alleviates ER stress and lessens programmed cell death
[44]. Recently, it is suggested that PDIs serve both specialized and overlapping functions to adapt to new biochemical needs or environments. Peng et al. [43] showed that transgenic tobacco overexpressing AtPDI6 was more tolerant to high concentrations of 2,4,6-Trichlorophenol (TCP) implying that AtPDI6 can be used for TCP detoxification by the way of overexpression in plants. In our experiments, two PDIs (PDIL6 A0A1U8GVQ2) and (PDIL1 A0A1U8GX36) showed higher abundance in the RC than in the SC without $B$. tabaci invasion. The level of PDIL1 increased more than 2-fold in the SB-SC but not in the RB-RC after B. tabaci invasion. All results suggest that the resistant peppers have strong basal defense through regulating ribosomal proteins and protein disulfide isomerases.

A well-defined response of plants to stress involves the enhanced production of heat shock proteins, which maintain the cellular proteostasis in limiting the production and accumulation of protein aggregates induced by stress, thereby contributing to restoring cellular protein homeostasis disrupted by stress conditions [45-47]. In the previous section, the functions of CPHsp70.2, HSP90 and Hsp70-1 has been discussed. Recently, it appears that some of these HSPs are capable of controlling the mRNA translation under normal or stress conditions $[47,48]$. Small HSPs and HSP101 are involved in the resolubilization of translation factors like eEF1B and eIF4A during the recovery phase $[46,47]$. Merret et al. [47] showed that HSP101 is required for the efficient release of ribosomal protein mRNAs from stress granules resulting in a rapid restoration of the translation machinery by producing new ribosomal proteins. In our study, A0A1U8G5J6, a chaperone protein ClpB1-like protein, homologous to Arabidopsis HSP101, increased 1.72-folds in response to B. tabaci attack in the RB but maintained no change in the SB (Table 1). These observations suggest that the resistant genotype can rapidly adjust translational levels following B. tabaci stress.

\section{Lipid metabolism-related proteins}

Lipid-mediated signal processes are crucial for cell survival, growth and differentiation and for plant responses to biotic and abiotic cues such as salinity, pests, and pathogens [49]. Signaling lipids include a wide range of lipid classes, such as lysophospholipid, fatty acid and phosphatidic acid. In the present study, AIMI (A0A1U8E9J9), a peroxisomal fatty acid beta-oxidation multifunctional protein, was increased in the RB. AIMI is essential for seedling establishment and is also involved in JA biosynthesis [50]. Phospholipase D (PLD) hydrolyzes common membrane phospholipids, to generate a free head group and phosphatidic acid (PA). The PLD-mediated hydrolysis of phospholipids is highly prominent in plants and play important roles in plant response to stress, including plant- 
pathogen interactions [51]. In our experiment, PLD alpha 1 (A0A1U8F9H1) and PLDrp1 (A0A1U8FRJ4) were not induced by $B$. tabaci attack in both genotypes, but the expression levels of them were higher in the $\mathrm{RC}$ than that in the SC (about 3.0-fold). Arabidopsis mutants with antisense suppression of PLD $\alpha$ expression decreased PA and JA production. PLD has been implicated in JA/oxylipin formation in plant interaction with Botrytis cinere and virulent Pst DC3000 [52]. The function of PLDrp1 remains unknown, but its abundant expression and distribution in plants suggest that it binds PA and acts downstream of PLD $\alpha$ pathway. The PA-binding phosphoprotein PLDrp1 is regulated by PLD 1 in a stressdependent manner [53]. Our results suggest that PLDmediated signals in SB help improve resistance against $B$. tabaci attack, though the expression of PLDrp1 and PLD $\alpha 1$ were not activated by $B$. tabaci.

JAs play a central and conserved role in promoting resistance to a broad spectrum of insects, which are lipidderived signals originating from $\alpha$-linolenic acid in chloroplast membranes [50]. Plant lipoxygenases (LOXs) catalyze the oxidation of polyunsaturated fatty acids, generating hydroperoxy fatty acids. LOX2 encodes a 13(S)-lipoxygenase (LOX), that control the first dedicated step in the biosynthesis of JAs, catalysing the initial step of $\alpha$-linolenic acid into (13S)-hydroperoxyoct adecatrienoic acid. In this study, the LOX2 (F2YL87), homologous to Arabidopsis AtLOX2 was increased significantly in the $\mathrm{SB}$, but not in the RB. In rice, overexpressing OsLOX plants increased endogenous levels of JA, showing reduced plant mortality when infested with the phloem-feeding brown plant hopper [54]. In maize, ZmLOX10, a 13-LOX was involved in resistance against chewing Spodoptera exigua larvae [55]. In barley, LOX2.2 overexpressing lines showed up-regulation of some other JA-regulated genes with lower aphid number, suggesting that LOX2.2 plays a role in the activation of JA-mediated responses and indicates the involvement of LOX2.2 in basic defense responses [56]. Recently, it is reported that LOX2 is involved in green leaf volatiles (GLVs) biosynthesis in Arabidopsis [57]. GLVs have been shown to induce defense responses and are involved in indirect defense in plant-insect interactions $[58,59]$. It is also reported that LOX2 is involved in singlet oxygen generation as a response to wounding induced by herbivore as well as by physical factors, which provide novel insight into wound-induced signaling in the local defense reaction [60]. Though LOX2 was only induced by $B$. tabaci in the SB, RC has a higher level of LOX2 compared with SC under normal conditions. The $B$. tabaci-regulated LOX2 expression was further confirmed by PRM and RT-qPCR (Fig. 6 and Fig. 7). Thus, the susceptible pepper plants seem to activate their basal defense mechanism of LOX2-mediated JA signal under
B. tabaci attack, which has already existed and maintained a high level in the resistant plants.

\section{DAPs involved in photosynthesis}

In addition to the herbivore-induced production of physical and chemical defenses, numerous changes in plant primary metabolism occur in response to insect herbivores [61]. Research on changes in primary metabolism associated with insect feeding has been focused largely on the role of carbohydrates as products of photosynthesis. In the present study, KEGG enrichment analysis has revealed that eight proteins (A0A1U8GUM8, A0A1U8FUM0, A0A1U8FRH4, A0A1U8GVK4, A0A1U 8FGM0, A0A1U8FJN4, A0A1U8FZN5 and A0A1U 8EAE0) are involved in photosynthesis system (Additional file 1: Figure $\mathrm{S} 1$ ) and seven proteins (A0A1U8FNB3, A0A1U8HDS6, A0A1U8E7W8, A0A1U 8GDS4, A0A1U8GZ15, A0A1U8HK56, A0A1U8FHQ4) are involved in carbon fixation in photosynthetic organisms (Additional file 2: Figure S2). Besides, A0A1U8FNB3, A0A1U8GZ15, O78327, A0A1U8E7H4, A0A1U8EJC2 and A0A1U8HFF2 were also involved in the photosynthetic $\mathrm{CO}_{2}$ fixation process. For example, Chloroplast TKL (transketolase, A0A1U8FNB3; O78327) is a key enzyme of plant carbon metabolism due to its amphibiotic role in both the Calvin-Benson-Bassham (CBB) cycle and the oxidative pentose phosphate pathway [62]. Interestingly, most of proteins' expression level in the RC were significantly higher than those in the SC, but these proteins were not significantly increased in the RB and SB (Table 1).

There are two conflicting views on how plants should alter photosynthesis, and thereby carbon fixation, to optimize defense. Photosynthetic activity might be promoted or be reduced by herbivory [61]. For example, Bilgin et al. [63] showed that biotic stress globally downregulates photosynthesis genes. While Halitschke et al. [64] showed herbivore-specific elicitation of photosynthesis by mirid bug salivary secretions in the wild tobacco. In this study, the idea that increased photosynthesis is highly resistant to $B$. tabaci was supported by photosynthesis-related protein expressions using iTRAQ method. Previously, wheat and barley resistance to Russian wheat aphids has been associated with increased expression of photosynthesis-related genes after B. tabaci attack $[65,66]$. Photosynthetic activity could promote because (1) synthesis of defensive metabolites requires carbon fixation or (2) increasing photosynthetic activity to compensate for the loss of leaf area by insects [61]. The results showed that the resistant material had high photosynthesis ability and can respond to $B$. tabaci attack, suggesting that photosynthesis and carbon metabolism might be involved in the resistance of pepper to B. tabaci. 
Some other proteins were also found involved in plant defense. For example, SEOR1 is a phloem filament protein involved in plant defense [67]. Aphids of the species Myzus persicae on Arabidopsis thaliana AtSEOR1 and AtSEOR2 mutants perform worse when compared to aphids on control plants, indicated by reduced reproduction and shortened reproduction period. However, Anstead et al. [68] concluded that SEOs were not involved in plant defense against phloem-feeding insects. Pagliari [69] showed that the low phytoplasma titer was found in AtSEOR1 mutant lines indicating the possible involvement of this gene in plant defense mechanism. Besides, a possible role of AtSEOR1-mediated JA and cis-12-oxo-phytodienoic acid metabolisms was observed in plant defense against phytoplasmas. PGK2, a nucleusencoded chloroplast phosphoglycerate kinase, plays a central role in cell metabolism. PGK protein is required for efficient watermelon mosaic virus (WMV, genus Pot virus) infection in the Arabidopsis [70].

\section{Conclusions}

In the present study, an overview of the protein expression profile in the pepper resistant genotype and sensitive genotype in response to $B$. tabaci attack at $48 \mathrm{~h}$ was first explored by iTRAQ technique. The proteomic data presented here will help us to further understand the molecular mechanisms of plant resistance to B. tabaci. It is suggested plant express more redox regulation-related proteins to deal with the oxidative damage caused by $B$. tabaci to improve plant tolerance. The PR3, Hsp70-1, HSP101 and JA pathway were more active in the resistant genotype, which might contribute to $B$. tabaci resistance in pepper. Meantime, our results support the view of increased photosynthesis (carbon metabolism) is involved in the resistant of pepper to B. tabaci. Besides, ANN4, CRT3, CEQORH and AIMI were specifically found here involved in $B$. tabaci-pepper interaction processes. In the future, studies on the function of specific proteins found in this study will be helpful to explore the mechanisms of host resistance to $B$. tabaci attack.

\section{Methods}

\section{Plant materials and insect maintenance}

In this study, the pepper (Capsicum annuum) genotype xinsujiao No.15 (resistant genotype, RG) and the genotype sujiao No.15 (susceptible genotype, SG) were acquired from the Vegetable Institute, Jiangsu Academy of Agricultural Sciences, Nanjing, China. Seeds were grown in a pest-free growth chamber at Yangzhou University, China. The plants were irrigated and fertilized according to horticultural practices but without spraying herbicides. A virus free colony of B. tabaci biotype B used for infestation was maintained on tomato in greenhouse of insectary.

\section{Non-preference test}

Two resistant pepper seedlings and two susceptible seedlings at 7 th leaf stage with approximately the similar leaf area were selected and placed in a 60-mesh gauze cage to prevent the whitefly escape. Four pots of seedlings were arranged in a circle. 200 adult insects were collected in a container and hung into the center of the circle. The number of adults on each seedling was counted $24 \mathrm{~h}, 48 \mathrm{~h}$ and $72 \mathrm{~h}$ after release, and the average number of insects settled on each variety was determined based on the number of insects on the 72-h seedling. The experiments were performed in 5 biological replicates and repeated twice to confirm the results.

\section{Egg hatchability}

For egg hatchability test, the 7th leaf stage healthy seedlings (cv. RG and SG) were selected. Each plant was covered with 60-mesh gauze and infested with five pairs of newly emerged adult $B$. tabaci. At 3 days after release, the eggs (including nymphs) in every seedling were counted. The experiments were performed in five biological replicates and repeated twice to confirm the results.

\section{B. tabaci infestation assay}

The tested samples (cv. RG and SG) were grown to the 7 th leaf stage. 50 adult insects were collected and released onto the 5th leaf and closed with a small ventilate pocket (Additional file 3: Figure S3). The plants grown as others without the insect infestation were set as control. After $B$. tabaci infestation for $48 \mathrm{~h}$, the infected leaves were cut and frozen immediately in liquid nitrogen, and store at $70{ }^{\circ} \mathrm{C}$ refrigerator for further use. All experiments were performed in three biological replicates.

\section{Protein extraction and reductive alkylation treatment}

The design of proteomic study is shown in Fig. 1. For protein extraction, twelve leaf samples (three biological replicates of two genotypes for the control and B. tabaci infestation; Treatment: SB and RB mean SG pepper and RG pepper with $B$. tabaci infestation, respectively; Control: SC and RC refer to SG pepper and RG pepper without B. tabaci infestation, respectively) were individually ground to powder in liquid nitrogen, and incubated in lysis buffer (7 M Urea, $2 \mathrm{M}$ Thiourea, 4\% SDS, $40 \mathrm{mM}$ Tris- $\mathrm{HCl}, \mathrm{pH} 8.5)$ containing $1 \mathrm{mM}$ PMSF and $2 \mathrm{mM}$ EDTA (final concentration) for $5 \mathrm{~min}$, then $10 \mathrm{mM}$ DTT (final concentration) were add to the sample. The samples were sonicated for $15 \mathrm{~min}$ and centrifuged at $4{ }^{\circ} \mathrm{C}$, $13,000 \times \mathrm{g}$ for $20 \mathrm{~min}$. The supernatant was transferred to a new tube and mixed with 4 volumes of precooled alkylation at $-20^{\circ} \mathrm{C}$ overnight. After centrifugation, the protein pellets were air-dried and resuspended in $8 \mathrm{M}$ urea/100 mM TEAB ( $\mathrm{pH} 8.0)$. Protein samples were 
reduced with $10 \mathrm{mM} \mathrm{DTT}$ at $56^{\circ} \mathrm{C}$ for $30 \mathrm{~min}$, and alkylated with $50 \mathrm{mM}$ iodoacetamide (IAM) for $30 \mathrm{~min}$ in the dark.

\section{Trypsin digestion and ITRAQ labeling}

After diluted 5 times with $100 \mathrm{mM}$ TEAB, $100 \mu \mathrm{g}$ of proteins from each sample were used for tryptic digestion. Trypsin (Promega, Madison, WI, USA) was added at an enzyme-protein ratio of $1: 30(\mathrm{w} / \mathrm{w})$, and digested at $37^{\circ} \mathrm{C}$ overnight. The digested peptides were acidified using equal volume of $0.1 \%$ formic acid (FA) solution and desalted with Strata-X C18 column. The acidified enzymatic hydrolysate was injected to column for three times, then washed the column with solvent A $(0.1 \%$ FA in $5 \% \mathrm{ACN})$ twice, the peptides were eluted $1 \mathrm{ml}$ with solvent B $(0.1 \%$ FA in $80 \%$ ACN). Finally, peptides were lyophilized and reconstituted in $20 \mathrm{ul} 0.5 \mathrm{M}$ TEAB for peptides labeling with iTRAQ 4-plex kits (AB Sciex Inc., USA) according manufacturer's protocol: Control and $B$. tabaci treatment of two pepper genotypes were labeled respectively as Additional file 11: Table S8 shown. The labeled samples were combined and lyophilized. Next, labeled samples were fractionated using highperformance liquid chromatography (HPLC) system (Thermo DINOEX Ultimate 3000 BioRS) with a Durashell C18 (5 um, $100 \AA, 4.6 \times 250 \mathrm{~mm}$ ) and 12 fractions collected.

\section{LC-ESI-MS/MS analysis}

Each fraction was dissolved in $30 \mu \mathrm{l}$ of $2 \%$ acetonitrile and analyzed using Triple TOF 5600+ mass spectrometer coupled with the Eksigent nanoLC System (SCIEX, USA). $5 \mathrm{ml}$ of peptide sample was loaded onto a C18 trap column $(5 \mu \mathrm{m}, 100 \mu \mathrm{m} \times 20 \mathrm{~mm})$, and eluted at 300 $\mathrm{nL} \cdot \mathrm{min}^{-1}$ onto a C18 analytical column $(3 \mu \mathrm{m}, 75 \mu \mathrm{m} \times$ $150 \mathrm{~mm}$ ) over a $90 \mathrm{~min}$ gradient. The two mobile phases were buffer A (2\% acetonitrile/0.1\% formic acid/98\% $\mathrm{H}_{2} \mathrm{O}$ ) and buffer B (98\% acetonitrile/0.1\% formic acid/ $2 \% \mathrm{H}_{2} \mathrm{O}$ ). For IDA (information dependent acquisition), survey scans were acquired in $250 \mathrm{~ms}$ and 30 production scans were collected in $100 \mathrm{~ms}$ per scan. MS1 spectra were collected in the range $350-1500 \mathrm{~m} \cdot \mathrm{z}^{-1}$, and MS2 spectra were collected in the range of $100-1500 \mathrm{~m} \cdot \mathrm{z}^{-1}$. Precursor ions were excluded from reselection for $15 \mathrm{~s}$.

\section{Data analysis}

Protein identification and quantification were performed by a search against the UniProt $C$. annuum protein database $(39,809$ proteins, update in Oct. 2017). Biological modifications were selected as ID focus. Bias Correction and Background Correction was checked for protein quantification and normalization. All identified proteins had an Unused Protscore of $>1.3$ (which corresponds to proteins identified with $>95 \%$ confidence), as calculated by the software and a global false discovery rate (FDR) of $\leq 1 \%$ determined at the protein level by the PSPEP algorithm. To be considered as being differentially expressed, proteins were required to have a $p$ value of $\leq 0.05$, as calculated by the software. For three biological replicates, the ratio of median expression between Case and Control was defined as fold changes. Statistical significance of the difference in the levels of expression of proteins between samples to be compared was determined by student's t-test (two-tailed and unpaired) to correct for multiple hypothesis testing. For protein abundance ratios measured using iTRAQ, a 1.50-fold or 0.67 -fold change threshold in the average value of three replicates with $P$-value $<0.05$ in at least two experiments were classified as a significant change.

\section{Bioinformatics}

In this study, the functional annotations of all differential abundance proteins (DAPs) were performed by using a localized Blast2go v2.6 against program against the NCBInr plant database (https:/github.com/wegnerce/ taxomias). The biological and functional properties of all the identified proteins were mapped with Gene Ontology (GO) Terms (http://geneontology.org/). The functional classification of the proteins using Clusters of Orthologous Groups of Proteins System (http://www. ncbi.nlm.nih.gov/COG/) was performed. All of the identified proteins were mapped to a pathway enrichment in the Kyoto Encyclopedia of Genes and Genomes (KEGG).

\section{Targeted protein quantification by parallel reaction monitoring (PRM)}

Twelve proteins including 2 reference proteins were selected for validation by PRM on Triple TOF $5600+$ LCMS/MS system (SCIEX). Protein extraction and tryptic digestion were performed in the same way as in the iTRAQ experiment. MS data acquisition was first performed in DDA mode to obtain MS/MS spectra for the 40 most abundant precursor ions following each survey MS1 scan in each cycle. Protein Pilot software was used to identify proteins, and the database searching results were brought into Skyline software for spectra library building. Target proteins for PRM validation were imported to the software Skyline, and the peptides for protein quantification were selected according to the ion signals in spectra library. A list of associated peptides containing $\mathrm{m} / \mathrm{z}$ values and retention times was exported from Skyline, and imported to MS control software Analyst for PRM acquisition method construction. PRM method was run against the biological samples of interest, evaluated and refined to develop the highest quality assay. Data collection of each sample was performed using the final PRM acquisition method on the qTOF mass spectrometer, where each precursor ion was 
selected by the quadrupole, fragmented, and then all fragment ions were quantified in the TOF mass analyzer. To eliminate protein carryover, a "blank" needed to be run between adjacent samples for column washing. Data processing was done in Skyline, and the quantification results were manually inspected for each peptide of the targeted proteins.

\section{Quantitative real-time PCR (qRT-PCR) analysis}

To investigate the correlation between transcript and protein level and confirm response proteins in the iTRAQ and PRM data set, 6 different expressional proteins were selected for further qRT-PCR analysis. Actin-97 and phosphoenolpyruvate carboxylase 2 were set as internal reference gene. The full-length cDNA sequence of the corresponding gene was obtained from NCBI based on the interested protein information, and primers (Additional file 12: Table S9) were designed using the NCBI primer tool. After $48 \mathrm{~h} \mathrm{~B}$. tabaci feeding, the total RNA was extracted from pepper leaves by Trizol extraction method as manufacturer's description (Invitrogen Trading (Shanghai) Co., Ltd), and reverse transcribed cDNA from equal amounts $(1.0 \mu \mathrm{g})$ of total RNA using the ReverTra Ace qPCR RT Kit (Toyobo life science Co.). The reactions were performed using SuperReal PreMix Plus kit (Toyobo life science Co.) according to the protocol. The thermal cycler was performed as follows: 1 cycle of $95^{\circ} \mathrm{C} 1 \mathrm{~min}$; followed by 40 cycles of $95^{\circ} \mathrm{C}, 15 \mathrm{~s}$ and $60^{\circ} \mathrm{C}, 30 \mathrm{~s}$. Relative gene expression was calculated by the $2^{-\Delta \Delta \mathrm{CT}}$ method. The experiment was repeated three times.

\section{Additional files}

Additional file 1: Figure S1. KEGG pathway of photosynthesis (ko00195). PsBo, A0A1U8FJN4/ A0A1U8FZN5; PsBp, A0A1U8EAE0; PsB27, A0A1U8FGM0; PsaD, A0A1U8GVK4; PetA, A0A1U8FRH4, PetH, A0A1U8FRH4; gamma, A0A1U8FUMO, b, A0A1U8GUM8 (TIF $4719 \mathrm{~kb}$ )

Additional file 2: Figure S2. KEGG pathway of carbon fixation in photosynthetic organisms (ko00710). 4.1.2.13, A0A1U8FHQ4; 2.2.1.1, 078327/ A0A1U8FNB3; 3.1.3.11, AOA1U8GDS4; 2.7.2.3, A0A1U8HDS6; 3.1.3.37, AOA1U8GZ15; 1.1.1.40, AOA1U8E7W8 (TIF $1471 \mathrm{~kb}$ )

Additional file 3: Figure S3. The controlled container. 50 B. tabaci adult insects were collected and released onto the 5th leaf and closed with a small pocket. (TIF $7155 \mathrm{~kb}$ )

Additional file 4: Table S1. Information statistics of total protein identified (XLSX $9 \mathrm{~kb}$ )

Additional file 5: Table S2. Gene ontology (GO) analysis of annotated proteins (XLSX $11 \mathrm{~kb}$ )

Additional file 6: Table S3. GO biological process enrichment DAPs (XLSX $9 \mathrm{~kb}$ )

Additional file 7: Table S4. GO molecular function enrichment of DAPs (XLSX $114 \mathrm{~kb})$

Additional file 8: Table S5. GO cellular component enrichment of DAPs (XLSX $46 \mathrm{~kb}$ )

Additional file 9: Table S6. Pathway enrichment of RB-RC (XLSX $17 \mathrm{~kb}$ )

Additional file 10: Table S7. Number of DAPs obtained by different analytical methods (XLSX $23 \mathrm{~kb}$ )
Additional file 11: Table S8. Parrelism between samples and iTRAQ labeling (XLSX $12 \mathrm{~kb}$ )

Additional file 12: Table S9. Primers for qRT-PCR (XLSX $12 \mathrm{~kb}$ )

\section{Abbreviations}

DAPs: differential abundance proteins; iTRAQ: isobaric tag for relative and absolute quantification; JA: jasmonic acid; PRM: parallel reaction monitoring; qRT-PCR: quantitative RT-PCR; RB: resistant genotype infested with B. tabaci; RC: RB control; RG: resistant genotype; SA: salicylic acid; SB: susceptible genotype infested with B. tabaci; SC: SB control; SG: susceptible genotype

\section{Acknowledgments}

Not Applicable.

\section{Authors' contributions}

$X X, F C$ and $H D$ conceived of and designed the study. JX, YH and HB conducted the experiments. SR and XY performed GRT-PCR analysis. XX and HD performed data analysis. HD and XX wrote the manuscript. FC supervised and complemented the writing. All authors have read and approved this manuscript.

\section{Funding}

This work was supported by the National Natural Science Foundation of China (Grant No. 31101092), China Postdoctoral Science Foundation funded project (2012 T50520), Jiangsu Science and Technology Support Program (BE2017347), Jiangsu Modern Agriculture (Vegetable) Industry System Innovation Team (SXGC[2017]303) and Development and application of ecological prevention and control technology for important outbreak pests of facility fruits vegetables (CX[15]1041). The founders did not play any roles in the design, analysis, and interpretation of this study or relevant data.

Availability of data and materials

The materials used during the current study will be freely available upon request to corresponding author: fczhou@yzu.edu.cn

Ethics approval and consent to participate

Not applicable.

Consent for publication

Not applicable.

\section{Competing interests}

The authors declare that they have no competing interests.

\section{Author details}

${ }^{1}$ Joint International Research Laboratory of Agriculture and Agri-Product Safety, College of Bioscience and Biotechnology, Yangzhou University, Yangzhou 225009, China. ${ }^{2}$ College of Horticulture and Plant Protection, Yangzhou University, Yangzhou 225009, China.

Received: 18 October 2018 Accepted: 24 May 2019

Published online: 21 June 2019

References

1. Barro PJD, Liu SS, Boykin LM, Dinsdale AB. Bemisia tabaci: a statement of species status. Annu Rev Entomol. 2009;56:1-19.

2. Dinsdale A, Cook L, Riginos C, Buckley YM, Barro PD. Refined global analysis of Bemisia tabaci (hemiptera: sternorrhyncha: aleyrodoidea) mitochondrial cytochrome oxidase 1 to identify species level genetic boundaries. Ann Entomol Soc Am. 2010;103:196-208.

3. Yang N, Wen X, Xin Y, Wang S, Wu Q, Li R, Pan HP, et al. Transcriptomic and proteomic responses of sweetpotato whitefly, Bemisia tabaci, to thiamethoxam. PLoS One. 2013;8:e61820.

4. Elsen OVD, Lucatti AF, Heusden SV, Broekgaarden C, Mumm R, Dicke M, et al. Quantitative resistance against Bemisia tabaci in Solanum pennellii: genetics and metabolomics. J Integr Plant Biol. 2016;58:397-412.

5. Raza A, Malik HJ, Shafiq M, Amin I, Scheffler JA, Scheffler BE, et al. RNA interference based approach to down regulate osmo regulators of whitefly (Bemisia tabaci): potential technology for the control of whitefly. PLoS One. 2016;11:e0153883. 
6. Yin H, Yan F, Ji J, Li Y, Wang R, Xu C. Proteomic analysis of Arabidopsis thaliana, leaves infested by tobacco whitefly Bemisia tabaci, (gennadius) B biotype. Plant Mol Biol Report. 2012;30:379-90.

7. Broekgaarden C, Snoeren TA, Dicke M, Vosman B. Exploiting natural variation to identify insect-resistance genes. Plant Biotechnol J. 2011;9: 819-25.

8. Zarate SI, Kempema LA, Walling LL. Silverleaf whitefly induces salicylic acid defenses and suppresses effectual jasmonic acid defenses. Plant Physiol. 2007;143:866-75

9. Zhang PJ, He YC, Zhao C, Ye ZH, Yu XP. Jasmonic acid-dependent defenses play a key role in defending tomato against Bemisia tabaci nymphs, but not adults. Front Plant Sci. 2018;9. https://doi.org/10.3389/fpls.2018.01065.

10. Zhang PJ, Wei JN, Zhao C, Zhang YF, Li CY, Liu SS, Dickee M, Yu XP, Turlingsf TCJ. Airborne host-plant manipulation by whiteflies via an inducible blend of plant volatiles. Proc Natl Acad Sci U S A. 2019. https:// doi.org/10.1073/pnas.1818599116

11. Wt VDV, Levesque CS, Perring TM, Walling LL. Local and systemic changes in squash gene expression in response to silverleaf whitefly feeding. Plant Cell. 2000;12:1409-23.

12. Puthoff DP, Holzer FM, Perring TM, Walling LL. Tomato pathogenesis-related protein genes are expressed in response to trialeurodes vaporariorum and Bemisia tabaci biotype B feeding. J Chem Ecol. 2010;36:1271-85.

13. Li J, Zhu L, Hull JJ, Liang S, Daniell H, et al. Transcriptome analysis reveals a comprehensive insect resistance response mechanism in cotton to infestation by the phloem feeding insect Bemisia tabaci (whitefly). Plant Biotechnol J. 2016;14:1956-75.

14. Ibrahim M, Yasmeen S, Zaman G, Li B, Al-Qurainy F, Athar HUR, et al. Protein profiling analysis of Gossypium hirsutum, (malvales: malvaceae) leaves infested by cotton whitefly Bemisia tabaci, (homoptera: aleyrodidae). Appl Entomol Zool. 2016:51:1-9.

15. Peterson AC, Russell JD, Bailey DJ, Westphall MS, Coon JJ. Parallel reaction monitoring for high resolution and high mass accuracy quantitative, targeted proteomics. Mol Cell Proteomics. 2012;11:1475-88.

16. Wang ZX, Shang P, Li QG, Wang LY, Chamba YZ, Zhang H, Zhang B, Wu CX iTRAQ-based proteomic analysis reveals key proteins affecting muscle growth and lipid deposition in pigs. Sci Rep. 2017;7:46717.

17. Zeng W, Sun Z, Cai Z, Chen H, Lai Z, Yang S, et al. Proteomic analysis by iTRAQ-MRM of soybean resistance to Lamprosema indicate. BMC Genomics. 2017;18:444.

18. Chen QZ, Guo WS, Feng LZ, Ye XZ, Xie WF, Huang XP, et al. Transcriptome and proteome analysis of eucalyptus infected with Calonectria pseudoreteaudii. J Proteome. 2015;115:117-31.

19. Su Y, Xu L, Wang Z, Peng Q, Yang Y, Chen Y, et al. Comparative proteomics reveals that central metabolism changes are associated with resistance against Sporisorium scitamineum in sugarcane. BMC Genomics. 2016;17:800.

20. Wei Z, Hu W, Lin Q, Cheng X, Tong M, Zhu L, et al. Understanding rice plant resistance to the Brown planthopper (Nilaparvata lugens): a proteomic approach. Proteomics. 2009;9:2798-808.

21. Maffei ME, Mithöfer A, Boland W. Before gene expression: early events in plant-insect interaction. Trends Plant Sci. 2007;12:310-6.

22. War AR, Paulraj MG, Ahmad T, Buhroo AA, Hussain B, lgnacimuthu S, et al. Mechanisms of plant defense against insect herbivores. Plant Signal Behav. 2012:7:1306-20

23. Leterrier M, Corpas FJ, Barroso JB, Sandalio LM, del Río LA. Peroxisomal monodehydroascorbate reductase genomic clone characterization and functional analysis under environmental stress conditions. Plant Physiol. 2005;138:2111-23.

24. Foyer $\mathrm{CH}$, Noctor $\mathrm{G}$. Ascorbate and glutathione: the heart of the redox hub. Plant Physiol. 2011;155:2-18.

25. Park SJ, Huang Y, Ayoubi P. Identification of expression profiles of sorghum genes in response to greenbug phloem-feeding using CDNA subtraction and microarray analysis. Planta. 2006;223:932-47.

26. Thompson GA, Goggin FL. Transcriptomics and functional genomics of plant defence induction by phloem-feeding insects. J Exp Bot. 2006:57: 755-66.

27. Curien G, Giustini C, Montillet JL, Mas-Y-Mas S, Cobessi D, Ferrer JL, et al. The chloroplast membrane associated ceQORH putative quinone oxidoreductase reduces long-chain, stress-related oxidized lipids. Phytochemistry. 2016;122:45-55.

28. Khan AA, Quigley JG. Control of intracellular heme levels: heme transporters and heme oxygenases. Biochim Biophys Acta. 2011;1813:668-82.
29. Lee HJ, Mochizuki N, Masuda T, Buckhout TJ. Disrupting the bimolecular binding of the haem-binding protein 5 (AtHBP5) to haem oxygenase 1 (HY1) leads to oxidative stress in Arabidopsis. J Exp Bot. 2012;63:5967-78.

30. Mittler R. Oxidative stress, antioxidants and stress tolerance. Trends Plant Sci. 2002; 7:405-10.

31. Pan $X$, Zhu B, Zhu H, Chen $Y$, Tian $H$, Luo $Y$, et al. iTRAQ protein profile analysis of tomato green-ripe mutant reveals new aspects critical for fruit ripening. J Proteome Res. 2014;13:1979.

32. Pulido P, Llamas E, Rodriguezconcepcion M. Both Hsp70 chaperone and Clp protease plastidial systems are required for protection against oxidative stress. Plant Signal Behav. 2017;12:e1290039.

33. Oh SE, Yeung C, Babaeirad R, Zhao R. Cosuppression of the chloroplast localized molecular chaperone HSP90.5 impairs plant development and chloroplast biogenesis in Arabidopsis. BMC Res Notes. 2014;7:643.

34. Jungkunz I, Link K, Vogel F, Voll LM, Sonnewald S, Sonnewald U. AtHSP7015-deficient Arabidopsis plants are characterized by reduced growth, a constitutive cytosolic protein response and enhanced resistance to TuMV. Plant J. 2011;66:983-95.

35. Chandrashekar N, Ali S, Grover A. Exploring expression patterns of PR-1, PR2, PR-3, and PR-12 like genes in Arabidopsis thaliana, upon Alternaria brassicae, inoculation. Biotech. 2018:8:230.

36. Sinha M, Singh RP, Kushwaha GS, lqbal N, Singh A, Kaushik S, et al. Current overview of allergens of plant pathogenesis related protein families. Sci World J. 2014;2014:543195.

37. Lim CW, Lee SC. Arabidopsis abscisic acid receptors play an important role in disease resistance. Plant Mol Biol. 2015:88:313-24.

38. Huh SM, Noh EK, Kim HG, Jeon BW, Bae K, Hu HC, et al. Arabidopsis annexins AnnAt1 and AnnAt4 interact with each other and regulate drought and salt stress responses. Plant Cell Physiol. 2010;51:1499-514.

39. Liao C, Zheng Y, Guo Y. MYB30 transcription factor regulates oxidative and heat stress responses through ANNEXIN-mediated cytosolic calcium signaling in Arabidopsis. New Phytol. 2017:216:163.

40. Qiu Y, Xi J, Du L, Poovaiah BW. The function of calreticulin in plant immunity. Plant Sign Behav. 2012:7:907-10.

41. Kim JH, Nguyen NH, Nguyen NT, Hong SW, Lee H. Loss of all three calreticulins, CRT1, CRT2, and CRT3, causes enhanced sensitivity to water stress in Arabidopsis. Plant Cell Rep. 2013;32:1843-53.

42. Wittenberg G, Levitan A, Klein T, Dangoor I, Keren N, Danon A. Knockdown of the Arabidopsis thaliana chloroplast protein disulfide isomerase 6 results in reduced levels of photo inhibition and increased D1 synthesis in high light. Plant J. 2014;78:1003-13.

43. Peng RH, Qiu J, Tian YS, Gao J, Han H, Fu XY, et al. Disulfide isomerase-like protein AtPdil1-2 is a good candidate for trichlorophenol phytodetoxification. Sci Rep. 2017;7:40130.

44. Lu DP, Christopher DA. Endoplasmic reticulum stress activates the expression of a sub-group of protein disulfide isomerase genes and AtbZIP60 modulates the response in Arabidopsis thaliana. Mol Genet Genomics. 2008;280:199-210

45. Basha $E_{1} O^{\prime}$ Neill $H$, Vierling E. Small heat shock proteins and a-crystallins: dynamic proteins with flexible functions. Trends Biochem Sci. 2012;37:106-17.

46. Mcloughlin F, Basha E, Fowler ME, Kim M, Bordowitz J, Katiyar-Agarwal S, et al. Class I and II small heat-shock proteins protect protein translation factors during heat stress. Plant Physiol. 2016:172:16.00536.

47. Merret R, Carpenier MC, Favory JJ, Picart C, Descombin J, Bousquetantonelli

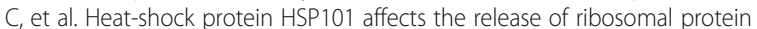
mRNAs for recovery after heat shock. Plant Physiol. 2017;174:1216-25.

48. Walters RW, Parker R. Coupling of ribostasis and proteostasis:Hsp70 proteins in mRNA metabolism. Trends Biochem Sci. 2015:40:552-9.

49. Wang X. Lipid signaling. Curr Opin Plant Biol. 2004;7:329-36.

50. Delker C, Zolman BK, Miersch O, Wasternack C. Jasmonate biosynthesis in Arabidopsis thaliana requires peroxisomal Beta-oxidation enzymes-additional proof by properties of PEX6 and AIM1. Phytochemistry. 2007;68:1642-50.

51. Okazaki $Y$, Saito K. Roles of lipids as signaling molecules and mitigators during stress response in plants. Plant J. 2014;79:584-96.

52. Zhao J, Devaiah SP, Wang C, Li M, Welti R, Wang X. Arabidopsis phospholipase Dbeta1 modulates defense responses to bacterial and fungal pathogens. New Phytol. 2013;199:228-40.

53. Ufer G, Gertzmann A, Gasulla F, Röhrig H, Bartels D. Identification and characterization of the phosphatidic acid-binding a. thaliana phosphoprotein PLDrp1 which is regulated by PLDa1 in a stress dependent manner. Plant J. 2017;92:276-90. 
54. Wang R, Shen W, Liu L, Jiang L, Liu Y, Su N, et al. A novel lipoxygenase gene from developing rice seeds confers dual position specificity and responds to wounding and insect attack. Plant Mol Biol. 2008;66:401-14.

55. Christensen SA, Nemchenko A, Borrego E, Murray I, Sobhy IS, Bosak L, et al. The maize lipoxygenase, ZmLOX10, mediates green leaf volatile, jasmonate and herbivore-induced plant volatile production for defense against insect attack. Plant J. 2013;74:59-73.

56. Losvik A, Beste L, Glinwood R, Ivarson E, Stephens J, Zhu LH, et al. Overexpression and down-regulation of barley lipoxygenase LOX2.2 affects jasmonate-regulated genes and aphid fecundity. Int J Mol Sci. 2017;18:2765.

57. Mochizuki S, Sugimoto K, Koeduka T, Matsui K. Arabidopsis lipoxygenase 2 is essential for formation of green leaf volatiles and five-carbon volatiles. FEBS Lett. 2016;590:1017-27.

58. Scala A, Allmann S, Mirabella R, Haring MA, Schuurink RC. Green leaf volatiles: a plant's multifunctional weapon against herbivores and pathogens. Int J Mol Sci. 2013;14:17781-811.

59. UI Hassan MN, Zainal Z, Ismail I. Green leaf volatiles: biosynthesis, biological functions and their applications in biotechnology. Plant Biotechnol J. 2015; 13:727-39.

60. Prasad A, Sedlářová M, Kale RS, Pospísil P. Lipoxygenase in singlet oxygen generation as a response to wounding: in vivo imaging in Arabidopsis thaliana. Sci Rep. 2017;7:9831.

61. Zhou S, Lou YR, Tzin V, Jander G. Alteration of plant primary metabolism in response to insect herbivory. Plant Physiol. 2015;169:1488-98.

62. Rocha AG, Mehlmer N, Stael S, Mair A, Parvin N, Chigri F, et al. Phosphorylation of Arabidopsis transketolase at Ser 428 provides a potential paradigm for the metabolic control of chloroplast carbon metabolism. Biochem J. 2014:458:313-22.

63. Bilgin DD, Zavala JA, Zhu J, Clough SJ, Ort DR, Delucia EH. Biotic stress globally downregulates photosynthesis genes. Plant Cell Environ. 2010;33: 1597-613.

64. Halitschke R, Hamilton JG, Kessler A. Herbivore-specific elicitation of photosynthesis by mirid bug salivary secretions in the wild tobacco Nicotiana attenuate. New Phytol. 2011;191:528-35.

65. Botha AM, Lacockvan L, Niekerk C, Matsioloko MT, Preez FB, Loots S, et al. Is photosynthetic transcriptional regulation in Triticum aestivum L. CV. 'TugelaDN' a contributing factor for tolerance to Diuraphis noxia (Homoptera: Aphididae). Plant Cell Rep. 2006;25:41-54.

66. Gutsche A, Heng-Moss T, Sarath G, Twigg P, Xia Y, Lu G, et al. Gene expression profiling of tolerant barley in response to Diuraphis noxia (Hemiptera: Aphididae) feeding. Bull Entomol Res. 2009;99:163-73.

67. Torsten W, Furch ACU, Zimmermann MR. How phloem-feeding insects face the challenge of phloem-located defenses. Front Plant Sci. 2013;4:336.

68. Anstead JA, Froelich DR, Knoblauch M, Thompson GA. Arabidopsis P-protein filament formation requires both AtSEOR1 and AtSEOR2. Plant Cell Physiol. 2012;53:1033-42

69. Pagliari L. On the role of phloem protein in plant-pathogen interaction; 2017

70. Ouibrahim L, Mazier M, Estevan J, Pagny G, Decroocq V, Desbiez C, et al. Cloning of the Arabidopsis RWM1 gene for resistance to watermelon mosaic virus points to a new function for natural virus resistance genes. Plant J. 2015;79:705-16.

\section{Publisher's Note}

Springer Nature remains neutral with regard to jurisdictional claims in published maps and institutional affiliations.

Ready to submit your research? Choose BMC and benefit from:

- fast, convenient online submission

- thorough peer review by experienced researchers in your field

- rapid publication on acceptance

- support for research data, including large and complex data types

- gold Open Access which fosters wider collaboration and increased citations

- maximum visibility for your research: over $100 \mathrm{M}$ website views per year

At BMC, research is always in progress.

Learn more biomedcentral.com/submissions 